\title{
Variability and distribution of the golden-headed weevil Compsus auricephalus (Say) (Curculionidae: Entiminae: Eustylini)
}

\author{
Jennifer C. Girón‡, M. Lourdes Chamorro§ \\ ‡ Natural Science Research Laboratory, Museum of Texas Tech University, Lubbock, United States of America \\ $\S$ Systematic Entomology Laboratory, ARS, USDA, c/o National Museum of Natural History, Smithsonian Institution, \\ Washington, DC, United States of America
}

Corresponding author: Jennifer C. Girón (entiminae@gmail.com)

Academic editor: Li Ren

Received: 15 Jun 2020 | Accepted: 03 Jul 2020 | Published: 09 Jul 2020

Citation: Girón JC, Chamorro ML (2020) Variability and distribution of the golden-headed weevil Compsus auricephalus (Say) (Curculionidae: Entiminae: Eustylini). Biodiversity Data Journal 8: e55474.

https://doi.org/10.3897/BDJ.8.e55474

\begin{abstract}
\section{Background}

The golden-headed weevil Compsus auricephalus is a native and fairly widespread species across the southern U.S.A. extending through Central America south to Panama. There are two recognised morphotypes of the species: the typical green form, with pink to cupreous head and part of the legs and the uniformly white to pale brown form. There are other Central and South American species of Compsus and related genera of similar appearance that make it challenging to provide accurate identifications of introduced species at ports of entry.
\end{abstract}

\section{New information}

Here, we re-describe the species, provide images of the habitus, miscellaneous morphological structures and male and female genitalia. We discuss the morphological variation of Compsus auricephalus across its distributional range, by revising and updating 
its distributional range, based on data from entomological collections in the U.S.A. and Canada. The revised distribution of $C$. auricephalus extends as far south as Zacapa in Guatemala. Records south from there correspond to a different species, with affinities to $C$. auricephalus that we discuss and illustrate. We also discuss morphological affinities and differences with other similar species. Furthermore, we summarise information regarding the biology, host plants and natural enemies of $C$. auricephalus.

\section{Keywords}

Broad-nosed weevils, native species, morphotypes, morphological variation, host plants, distribution

\section{Introduction}

Broad-nosed weevils of the subfamily Entiminae Schönherr, 1823 (Schönherr 1823) are amongst the most diverse groups of beetles worldwide. Entimines are known for their polyphagous feeding habits (Marvaldi et al. 2014) and include some of the, arguably, most stunning and charismatic forms of weevils: the genus Eupholus Boisduval, 1835 (Boisduval 1835) (known as "smurf-weevils", see Van Dam et al. 2017), the genus Pachyrhynchus Germar, 1824 (Germar 1824; for example, Háva and Rukmane 2018) and the genus Briarius Fischer de Waldheim, 1829 (Fischer de Waldheim 1829) (formerly Lamprocyphus Marshall, 1922 (Marshall 1922) (see del Río and Lanteri 2003).

There are 55 tribes recognised within Entiminae (Alonso-Zarazaga and Lyal 1999), most of them distributed in particular biogeographic regions of the world (Marvaldi et al. 2014). The tribe Eustylini Lacordaire, 1863 (Lacordaire 1863) is restricted, for the most part, to the Neotropical region, grouping 23 genera and 334 described species (Franz 2012). Eustylines are amongst the most commonly found broad-nosed weevils in Northern South America (e.g. Girón and Cardona-Duque 2018) and include many relatively large ( 7 to 25 $\mathrm{mm}$ ) and strikingly coloured species. Some eustylines are known as serious agricultural pests, such as the infamous "diaprepres root weevil", Diaprepes abbreviatus (Linnaeus, 1758) (Linnaeus 1758), which attacks the roots and foliage of citrus, sugar cane, coffee and other cultivated plants throughout the Caribbean region and Florida (Hall 1995, Simpson et al. 1996, Weissling et al. 1998, Wolcott 1922). Only in Puerto Rico, D. abbreviatus feeds on the foliage of over 40 plant species (Martorell 1945). "It is estimated that Diaprepes abbreviatus causes about 70 million dollars in damage annually in Florida. Estimates show the weevil infests more than 100,000 acres of citrus" (Weissling et al. 1998).

Species identification of Eustylini is quite challenging, partly because of their diversity and the lack of revisionary (and comprehensive) taxonomic studies for the group, but also because of the high degree of overlapping characters amongst eustyline genera. Furthermore, the limits of Eustylini, as a tribe, are still not clearly defined, as some genera in the tribe Geonemini Gistel, 1848 (Gistel 1848) exhibit quite similar morphologies and 
have been shown to cluster together with eustylines in phylogenetic analyses (Franz 2012, Marvaldi et al. 2018). Indeed, recent studies in Eustylini have referred to the "Exophthalmus genus complex" (Franz 2012, Zhang and Franz 2015, Zhang et al. 2017), as a mainly Caribbean and Central American group, including the genera Compsus Schönherr, 1823 (Schönherr 1823), Diaprepes Schöenherr, 1823 (Schönherr 1823), Eustylus Schönherr, 1842 (Schönherr 1842), Exophthalmus Schönherr, 1823 (Schönherr 1823), Exorides Pascoe, 1881 (Pascoe 1881) (all Eustylini) and Lachnopus Schönherr, 1840 (Schönherr 1840) (Geonemini), amongst which, at certain points, the generic limits are indistinct. A similar situation occurs amongst the genera Compsus, Brachyomus Lacordaire, 1863 (Lacordaire 1863), Chauliopleurus Champion, 1911 (Champion 1911), Exorides, Eustylus, Oxyderces Schönherr, 1823 (Schönherr 1823) and Xestogaster Marshall, 1922 (Marshall 1922), here called the "Compsus genus complex", which includes nearly 180 species distributed almost exclusively throughout South America (O'Brien and Wibmer 1982, Wibmer and O'Brien 1986, Morrone 1999). The majority of species described in these genera remain only known from their original descriptions, have never been illustrated and only rarely those descriptions include information about the genitalia. Some geonemines, like Epicaerus Schönherr, 1834 (Schönherr 1834) and Compsonomus Jekel, 1875 (Jekel 1875), exhibit morphological similarities with members of the "Compsus genus complex".

The taxonomic confusion of the Eustylini became painfully evident with recent domestic and port interceptions in the U.S.A. of an unknown, colourful and eye-catching Colombian eustyline species loosely associated with imported cut flowers (L. Chamorro, pers. obs.). This eustyline species was tentatively identified in the taxonomically confused and diverse Compsus genus complex, but further identification required comparisons with type material deposited in European institutions.

A single species in the Compsus genus complex occurs in the U.S.A., the golden-headed weevil, Compsus auricephalus (Say, 1824) (Say 1824) (Poole et al. 1996). This species ranges as far north as Illinois, west to Colorado and Arizona, east to Georgia and throughout Central America as far south as Panama (Wibmer and O'Brien 1986, Morrone 1999); in addition, it has been intercepted at ports in Ontario, Canada (McNamara 1991). Within this range, $C$. auricephalus exhibits two main colour morphs: predominantly green with pink/coppery head and part of the legs or completely white to pale brown (see Fig. 1). Champion (1911) highlighted that specimens from southern populations (e.g. Mexico (Acapulco), Guatemala, Costa Rica, Nicaragua, Panama) had a more elongate body, produced and acuminate elytral apices and strongly costate alternating interstriae. In addition to variation in colour, the species exhibits broad size variation $(8-15 \mathrm{~mm})$, with some males being half the size of the females.

Even though $C$. auricephalus can be regarded as a highly variable species, being the only species of the genus present in the U.S.A. and recognising its variation is probably good enough to differentiate it from other U.S.A. entimines. The problem is that the genus currently has over a hundred species across its distributional range, with some of them looking quite similar to either or both of the colour morphs of $C$. auricephalus. This situation may lead to misidentifications, especially of Central American specimens, causing 
problems at ports of entry given the current inability of determining if intercepted specimens belong to a native or exotic species.
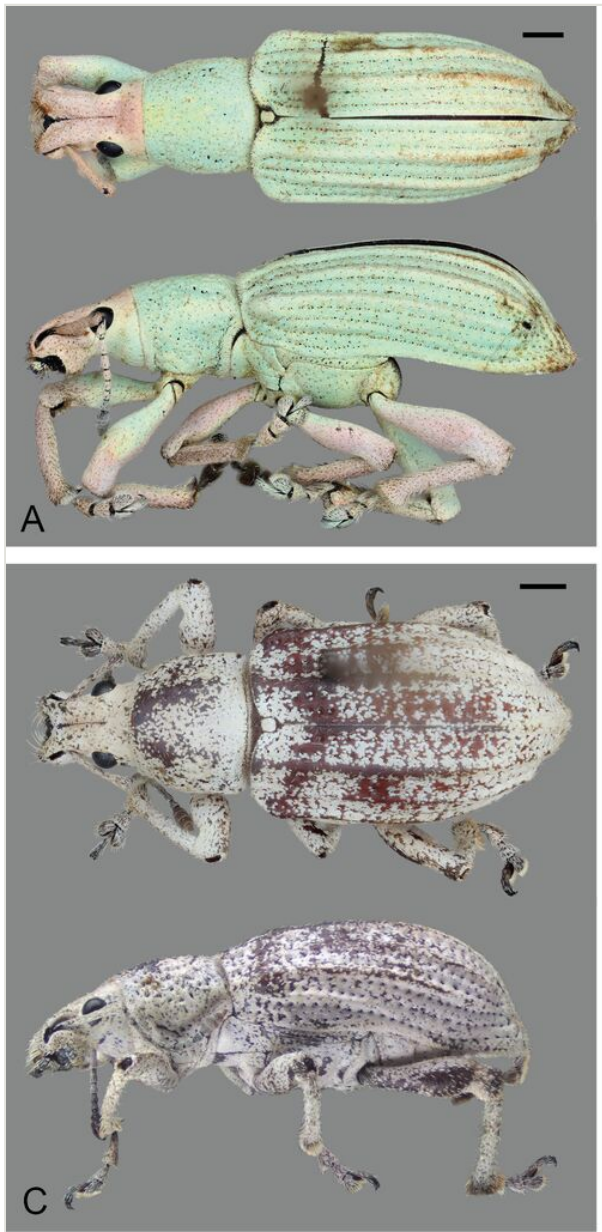
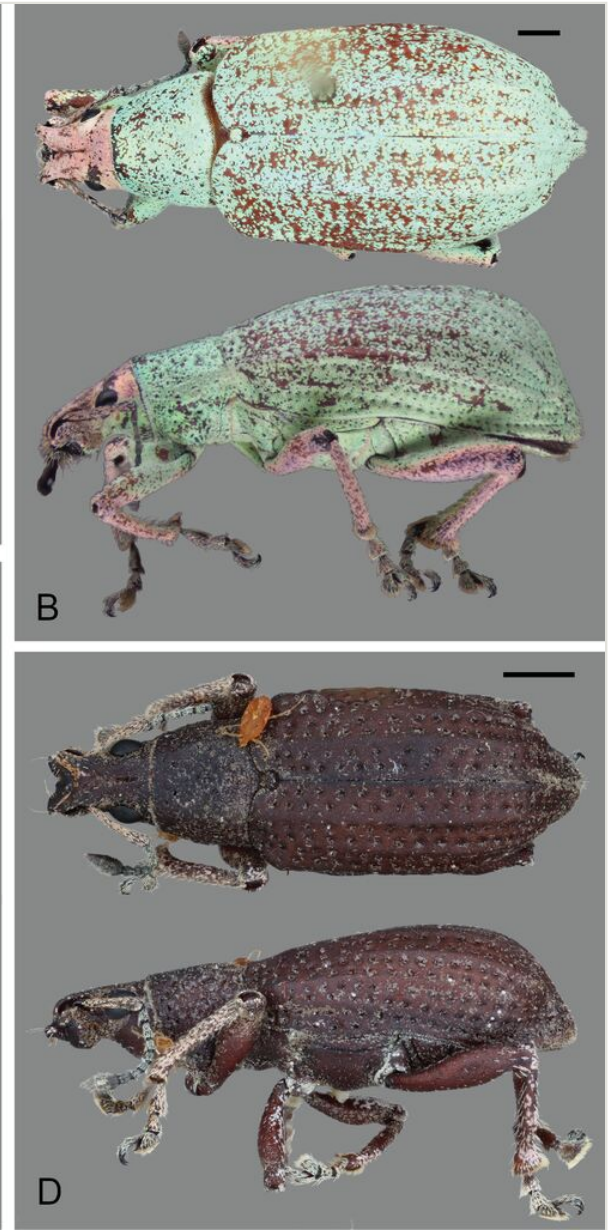

Figure 1. doi

Habitus and variation of Compsus auricephalus: (A) Mexico, Veracruz (CWOC0047) dorsal, lateral, (B) Mississippi, Washington County (CWOC0045) dorsal, lateral, (C) Texas, Brooks County (CWOC0002) dorsal, lateral, (D) Mississippi, Washington County (CWOC0814) dorsal, lateral. Scale bars: $1 \mathrm{~mm}$.

This study aims to clarify the status of Compsus auricephalus by (1) presenting a full redescription of the species with images representing its morphological characteristics and range of variation, (2) comparing $C$. auricephalus to externally similar species, (3) updating distributional records including a map and (4) presenting a summary of biological information including a list of host plants and natural enemies for the species, with a discussion on the status of $C$. auricephalus as a crop pest. The mouthparts, hind wings and male and female genitalia are described here for the first time. 


\section{Materials and methods}

\section{Specimen data}

Over 700 specimens were directly examined (Suppl. material 1 in addition to specimens listed under Materials below). Type material of miscellaneous species in the Compsus genus complex were examined in order to differentiate $C$. auricephalus from similarlylooking species. Examined specimens are deposited at the following institutions:

- $\quad$ ASUCOB Charles W. O'Brien Collection, Arizona State University, Tempe, Arizona (Arizona State University Biocollections 2019; Charles O’Brien, Emmy Engaser, Sangmi Lee, Nico Franz).

- $\quad$ CMNC Canadian Museum of Nature, Ottawa, Canada (Robert Anderson).

- MNHN Muséum National d'Histoire Naturelle, Paris, France (Hélène Perrin and Antoine Mantilleri).

- $\quad$ NHMUK The Natural History Museum [formerly British Museum (Natural History)], London, United Kingdom (Maxwell Barclay).

- $\quad$ NHRS Naturhistoriska Riksmuseet, Stockholm, Sweden (Johannes Bergsten).

- $\quad$ OSUC C.A. Triplehorn Insect Collection, Ohio State University, Columbus, Ohio (Johnson and Cora 2018; Luciana Musetti and Norman Johnson).

- $\quad$ TAMUIC Texas A \& M University, College Station, Texas (Texas A\&M University Insect Collection 2020; Karen Wright).

- $\quad$ TTUZ Texas Tech University, Lubbock, Texas (Museum of Texas Tech University (TTU) 2020; Jennifer Girón).

- USNM U.S. National Museum of Natural History, Smithsonian Institution, Washington, DC. (Lourdes Chamorro and Floyd Shockley).

- ZMUK Zoological Museum of Kiel University, Kiel, Germany (Michael Kuhlmann) loan initiated by ZMUC Natural History Museum of Denmark (University of Copenhagen, Zoological Museum, Copenhagen, Denmark (Alexey Solodovnikov).

Data for ASUCOB (part), CMNC, NHMUK and USNM were compiled into a DarwinCore file (Suppl. material 1). In addition, preserved specimen data were retrieved via SCAN (SCAN 2020) from the following collections:

- $\quad$ ASUHIC Hasbrouck Insect Collection, Arizona State University, Tempe, Arizona (Arizona State University Biocollections 2020).

- $\quad$ AUEM Auburn University Museum of Natural History Entomology, Auburn University, Auburn, Alabama (Callahan 2019).

- CASM Museum of Natural History, Chicago Academy of Sciences, Chicago, Illinois (Roberts 2020).

- MSUC Michigan State University, East Lansing, Michigan (A.J. Cook Arthropod Research Collection 2020).

- UAIC University of Arizona Insect Collection, University of Arizona, Tucson, Arizona (University of Arizona Insect Collection 2020).

- UCFC Stuart M. Fullerton Collection of Arthropods, University of Central Florida, Orlando, Florida (Song and Johnson 2018). 
- UMNH Utah Museum of Natural History, University of Utah, Salt Lake City, Utah (Natural History Museum of Utah (UMNH) 2020).

These datasets were also recovered through GBIF (GBIF.org 2020). Additional GBIF records (GBIF.org 2020) were recovered from the following datasets:

- Base de datos de la Colección zoológica del Instituto de Investigación de zonas desérticas de la Universidad Autónoma de San Luis Potosí (BDCZIID-UASLP) (Martínez de la Vega $\mathrm{G}$ and Comisión nacional para el conocimiento y uso de la biodiversidad 2018).

- Captura de datos de la Colección de Curculionoidea (Insecta: Coleoptera) de la Universidad Autónoma de Querétaro (Jones RW and Comisión nacional para el conocimiento y uso de la biodiversidad 2018).

- Computarización de la Colección Nacional de insectos Dr. Alfredo Barrera Marín del Museo de Historia Natural de la Ciudad de México (Díaz Batres ME and Comisión nacional para el conocimiento y uso de la biodiversidad 2018).

- Computarización de la colección científica del proyecto de control biológico de malezas de CSIRO-Australia (Segura Ponce de León 2020Segura Ponce de León, $\mathrm{R}$ and Comisión nacional para el conocimiento y uso de la biodiversidad C 2020).

- Elaboración de la base de datos de los ejemplares de la colección general de insectos adultos de la Dirección General de Sanidad Vegetal (Vega Ortiz, H E and Comisión nacional para el conocimiento y uso de la biodiversidad C 2020).

The dataset was recovered from GBIF on 5 June 2020, contains 728 occurrence records and can be downloaded from https://doi.org/10.15468/dl.rat633. 'Human observations' from iNaturalist (iNaturalist.org 2020; as opposed to 'preserved specimens', see Darwin Core terminology http://rs.tdwg.org/dwc/terms.htm) and Bugguide (VanDyk et al. 2020), as recovered from GBIF, were verified and included in our dataset, representing $124(17 \%)$ of the GBIF records. Only two records, both based on human observations, (Oak Mountain State Park, Shelby County, Alabama [https://bugguide.net/node/view/1694325] and Fondes Amandes Rd, Port of Spain, Trinidad and Tobago [https://www.inaturalist.org/observations/ 32103921]) were excluded given that the identity of the specimens cannot be confirmed from the pictures provided.

For the following collections, specimen data were obtained directly from collection's curators and compiled into our DarwinCore file (Suppl. material 1):

- $\quad$ CLEV Cleveland Museum of Natural History, Columbus, Ohio (Nicole Gunter).

- $\quad$ CMNC Canadian Museum of Nature, Ottawa, Canada (Robert Anderson).

- INHS Illinois Natural History Survey, Champaign, Illinois (Tommy McElrath, Dmitriev 2015).

- LSAM Louisiana State Arthropod Museum, Louisiana State University, Baton Rouge, Louisiana (Victoria Bayless, Nathan Lord).

- $\quad$ MEM Mississippi State University, Mississippi, Mississippi (Terence Schiefer). 
In total, 1606 specimen records are included in this study. Part of the coordinates presented in Suppl. material 1 (also available at https://doi.org/10.15468/chpymx) were obtained by approximating the locality data via Google Maps (https://www.google.com/ maps). The distribution map was created using SimpleMappr (Shorthouse 2010). Collection codes, listed here, correspond to those listed in Evenhuis 2020. Information on host plants was obtained both from literature records and label data.

\section{Morphological methods}

Specimens were examined using an AmScope SM-1TSZZ-144S stereomicroscope (magnification: 3.5X-180X) and a Zeiss Discovery v8. Genitalia dissections were prepared by removing the entire abdomen from the specimen and opening it along one side; then the abdomen was submerged in a solution of $10 \%$ potassium hydroxide $(\mathrm{KOH})$ and heated to $50^{\circ} \mathrm{C}$ overnight. Afterwards, the macerated abdomen was submerged in glacial acetic acid for 10 minutes and then rinsed with distilled water. Dissections were ultimately performed by placing the cleared abdomen on a microscope slide with a drop of glycerine.

Habitus photographs were taken with a Visionary Digital Passport II imaging system (Visionary Digital, Los Angeles, CA), using a Canon MP-E 65mm lens f/1:2.8 1-5X macro lens mounted on a Canon 5D Mark III camera body and the Macropod Pro 3D system using Canon MP-E 65mm lens f/2.8 1-5x macro lens on a Canon 6D camera body on Focus stacking rails controlled by StackShot and Canon MT-24EX Macro Twin Lite flash units (Macroscopic Solutions, Connecticut, USA). Images of internal structures were produced by stacking images taken through a Canon EOS 5D Mark II camera attached to an AmScope SM-1TSZZ-144S stereomicroscope or a Nikon Optiphot microscope at 100x magnification. The serial images were processed using Helicon Focus 5.3 software (Helicon Soft Ltd 2013) and Zerene Stacker (Zerene Systems LLC 2019).

The species re-description follows Franz (2010), for the most part and is based on a series of specimens examined. Morphological terms are in agreement with Torre-Bueno (Nichols 1989) and Lawrence and Ślipiński (2013), with additional terms for structures of the mouthparts (Ting 1936), metendosternite (Velázquez de Castro 1998), wings (Zherikhin and Gratshev 1995) and the male (Wanat 2007, Boudinot 2018, Génier 2019) and female genitalia (Howden 1995, Gaiger and Vanin 2008).

\section{Taxon treatment}

\section{Compsus auricephalus (Say, 1824)}

- $\quad$ BHL https://www.biodiversitylibrary.org/page/24668962

- $\quad$ GBIF https://www.gbif.org/species/5015904 


\section{Nomenclature}

\section{Taxonomy}

Family Curculionidae Latreille, 1802 (Latreille 1802)

Subfamily Entiminae Schönherr, 1823 (Schönherr 1823)

Tribe Eustylini Lacordaire, 1863 (Lacordaire 1863)

Genus Compsus Schönherr, 1823: 1140 (Schönherr 1823)

Type species: Curculio elegans Olivier, 1807: 328 (Olivier 1807; =Curculio argyreus Linnaeus, 1758: 384 (Linnaeus 1758)); by monotypy

\section{Compsus auricephalus (Say, 1824)}

Curculio auricephalus Say, 1824: 310 (Say 1824)

Platyomus auricephalus (Boheman 1833: 645) (new combination in Schönherr 1833)

Platyomus auriceps Schönherr, 1840: 183 (Schönherr 1840, Fig. 2)

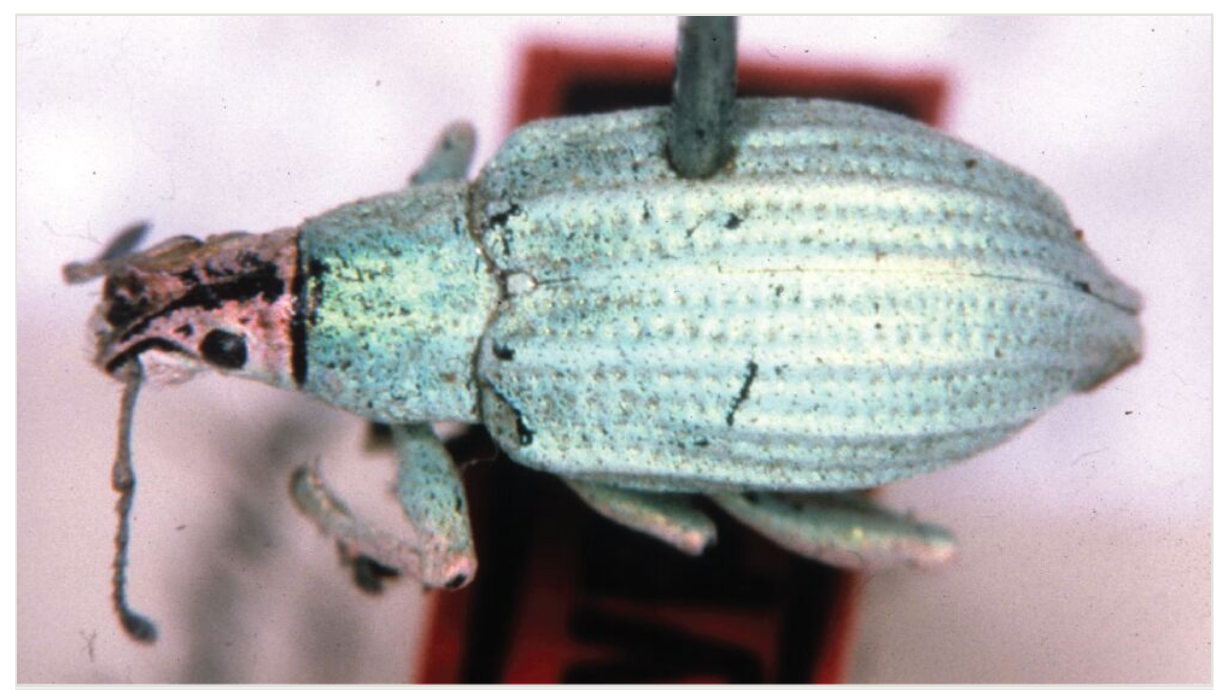

Figure 2. doi

Holotype of Platyomus auriceps Schönherr, 1840. Courtesy of M. Guadalupe del Río, taken at NHRS.

Say's original description for C. auricephalus (Say 1824) is quite brief and focuses on the colouration (of the green form) and sculpture of the species, represented by specimens collected in Mississippi and Missouri. For information and comments on the type material, see Prena (2018). In 1833, Boheman re-described the species in more detail, again based on the green form from Mississippi, under the name of Platyomus 
auricephalus (Schönherr 1833). The species was listed as Platyomus auriceps by Schönherr in 1840, recorded from Mexico as well (Schönherr 1840). LeConte and Horn (1876) offered a brief, but detailed description of the species, indicating its colour variation ranging from pale green with cupreous head and legs to white or pale brown, rather abundantly occurring from Georgia to Mexico and to the north in Colorado. Champion (1911) highlighted the great variation in size and colouration in C. auricephalus, attributing the colour variation to discolouration and comparing this to the situation of C. isabellinus (Boheman, 1833 in Schönherr 1833). At the same time, Champion extended the distributional range of $C$. auricephalus to Guatemala, Nicaragua, Costa Rica and Panama, indicating morphological variation in the southern populations of the species. Blatchley and Leng (1916) presented a brief diagnosis, with notes on their biology taken from Pierce (1916).

\section{Materials}

a. scientificName: Compsus auricephalus Say, 1824; taxonomicStatus: ACCEPTED; taxonID: 8801; kingdom: Animalia; phylum: Arthropoda; class: Insecta; order: Coleoptera; family: Curculionidae; taxonRank: SPECIES; genus: Compsus; specificEpithet: auricephalus; country: United States of America; countryCode: US; stateProvince: Louisiana; county: Saint Landry Parish; locality: Thistlethwaite National Wildlife Refuge; decimalLatitude: 30.669309; decimalLongitude: -92.022851; geodeticDatum: WGS84; georeferencedBy: Andrew Jansen; georeferenceSources: GeoLocate; georeferenceVerificationStatus: requires verification; eventDate: 1989-05-07T00:00:00; startDayOfYear: 127; year: 1989; month: 5; day: 7; individualCount: 1; catalogNumber: ASUHIC0019442; occurrenceDetails: http://api.gbif.org/v1/occurrence/search? occurrenceld=6cc2275b-b2cd-4ce8-ad3e-dec883a2ed72; recordedBy: D.A. Rider; identifiedBy: Charles W. O'Brien; dateldentified: 2011-01-01T00:00:00; modified: 2019-06-07T10:46:31.000+0000; rights: http://creativecommons.org/publicdomain/zero/ 1.0/; collectionID: 98d9b8ed-08d6-47fc-b324-2853e44d75d1; institutionCode: ASU; collectionCode: ASUHIC; basisOfRecord: PRESERVED_SPECIMEN; occurrenceID: 6cc2275b-b2cd-4ce8-ad3e-dec883a2ed72

b. ScientificName: Compsus auricephalus Say, 1824; taxonomicStatus: ACCEPTED; taxonID: 8801; kingdom: Animalia; phylum: Arthropoda; class: Insecta; order: Coleoptera; family: Curculionidae; taxonRank: SPECIES; genus: Compsus; specificEpithet: auricephalus; country: United States of America; countryCode: US; stateProvince: Texas; county: Cameron County; locality: Sabal Palm Grove Sanctuary; decimalLatitude: 25.8525; decimalLongitude: -97.4175; geodeticDatum: WGS84; georeferencedBy: Andrew Jansen; georeferenceSources: SCAN; georeferenceVerificationStatus: requires verification; eventDate: 1986-03-28T00:00:00; startDayOfYear: 87; year: 1986; month: 3; day: 28; individualCount: 1; catalogNumber: ASUHIC0019443; occurrenceDetails: http:// api.gbif.org/v1/occurrence/search?occurrenceld=b1 bb5a9c-1a79-4fc8-b570e7848a1fd401; recordedBy: E.G. Riley; identifiedBy: Charles W. O'Brien; dateldentified: 2006-01-01T00:00:00; modified: 2019-06-07T10:46:31.000+0000; rights: http:// creativecommons.org/publicdomain/zero/1.0/; collectionID: 98d9b8ed-08d6-47fcb324-2853e44d75d1; institutionCode: ASU; collectionCode: ASUHIC; basisOfRecord: PRESERVED_SPECIMEN; occurrenceID: b1bb5a9c-1a79-4fc8-b570-e7848a1fd401

c. scientificName: Compsus auricephalus Say, 1824; taxonomicStatus: ACCEPTED; taxonID: 8801; kingdom: Animalia; phylum: Arthropoda; class: Insecta; order: Coleoptera; family: Curculionidae; taxonRank: SPECIES; genus: Compsus; specificEpithet: 
auricephalus; country: United States of America; countryCode: US; stateProvince: Texas; county: Lubbock; locality: 2 Mi. N of Lubbock; decimalLatitude: 33.66685;

decimalLongitude: -101.83692; geodeticDatum: WGS84; coordinateUncertaintyInMeters: 1891; eventDate: 1980-06-06T00:00:00; startDayOfYear: 158; year: 1980; month: 6; day: 6; individualCount: 1; lifeStage: ADULT; preparations: dry pinned; catalogNumber: TTUZ_048376; occurrenceDetails: http://api.gbif.org/v1/occurrence/search? occurrenceld=d1a02bff-93a7-4746-8a40-829ac1a4e359; recordedBy: Bob Starkey; otherCatalogNumbers: TTU1997-058; identifiedBy: J. Girón; dateldentified: 2020-01-01T00:00:00; modified: 2015-01-08T00:00:00.000+0000; rights: http:// creativecommons.org/licenses/by-nc/3.0/; rightsHolder: Museum of Texas Tech University; accessRights: CC BY-NC (Attribution-Non-Commercial); collectionID: d4e788b4-5d52-47a3-873e-227c6df49c7b; institutionCode: TTU; collectionCode: TTU-Z; basisOfRecord: PRESERVED_SPECIMEN; occurrenceID: d1a02bff-93a7-4746-8a40-829ac1a4e359

d. scientificName: Compsus auricephalus Say, 1824; taxonomicStatus: ACCEPTED; taxonID: 8801; kingdom: Animalia; phylum: Arthropoda; class: Insecta; order: Coleoptera; family: Curculionidae; taxonRank: SPECIES; genus: Compsus; specificEpithet: auricephalus; country: Mexico; countryCode: MX; stateProvince: Tamaulipas; locality: 3 miles North of Cuidad Victoria; verbatimElevation: 800; decimalLatitude: 31.044186; decimalLongitude: -112.103; geodeticDatum: WGS84; coordinateUncertaintyInMeters: 6954; georeferencedBy: Alex Gregg (2014-08-14 13:58:34); georeferenceSources: georef batch tool 2014-08-14; GeoLocate; georeferenceVerificationStatus: reviewed - high confidence; eventDate: 1971-06-24T00:00:00; startDayOfYear: 175; year: 1971; month: 6; day: 24; verbatimEventDate: VI-24-1971; individualCount: 1; catalogNumber: TTUZ_213647; occurrenceDetails: http://api.gbif.org/v1/occurrence/search? occurrenceld=4832064b-a8ab-4fc5-b475-c15a59db588e; recordNumber: 13647; recordedBy: Ward; Brothers; otherCatalogNumbers: TTU1997-058; identifiedBy: C.W. O'Brien; modified: 2015-01-08T00:00:00.000+0000; rights: http://creativecommons.org/ licenses/by-nc/3.0/; rightsHolder: Museum of Texas Tech University; accessRights: CC BY-NC (Attribution-Non-Commercial); collectionID:

d4e788b4-5d52-47a3-873e-227c6df49c7b; institutionCode: TTU; collectionCode: TTU-Z; basisOfRecord: PRESERVED_SPECIMEN; occurrenceID: 4832064b-a8ab-4fc5-b475c15a59db588e

e. ScientificName: Compsus auricephalus Say, 1824; taxonomicStatus: ACCEPTED; taxonID: 8801; kingdom: Animalia; phylum: Arthropoda; class: Insecta; order: Coleoptera; family: Curculionidae; taxonRank: SPECIES; genus: Compsus; specificEpithet:

auricephalus; country: United States of America; countryCode: US; stateProvince: Texas; county: Starr; decimalLatitude: 26.562153; decimalLongitude: -98.7384; geodeticDatum: WGS84; coordinateUncertaintyInMeters: 53416; georeferenceRemarks: 88 High STARR COUNTY; eventDate: 1968-04-09T00:00:00; startDayOfYear: 100; year: 1968; month: 4; day: 9; verbatimEventDate: 9 IV 1968; habitat: Prosopis glandulosa; individualCount: 1; preparations: pinned; catalogNumber: TTU-Z_219309; occurrenceDetails: http:// api.gbif.org/v1/occurrence/search?occurrenceld=806e4d86-48ad-48d4af87-67227271b55a; recordedBy: R. R. Rogers; otherCatalogNumbers: TTU1997-058; identifiedBy: D. R. Whitehead; modified: 2015-01-08T00:00:00.000+0000; rights: http:// creativecommons.org/licenses/by-nc/3.0/; rightsHolder: Museum of Texas Tech University; accessRights: CC BY-NC (Attribution-Non-Commercial); collectionID: d4e788b4-5d52-47a3-873e-227c6df49c7b; institutionCode: TTU; collectionCode: TTU-Z; basisOfRecord: PRESERVED_SPECIMEN; occurrenceID: 806e4d86-48ad-48d4af87-67227271b55a 
f. scientificName: Compsus auricephalus Say, 1824; taxonomicStatus: ACCEPTED; taxonID: 8801; kingdom: Animalia; phylum: Arthropoda; class: Insecta; order: Coleoptera; family: Curculionidae; taxonRank: SPECIES; genus: Compsus; specificEpithet: auricephalus; country: United States of America; countryCode: US; stateProvince: Texas; county: Hall; locality: Estelline; decimalLatitude: 34.54667 ; decimalLongitude: -100.43778; geodeticDatum: WGS84; coordinateUncertaintylnMeters: 1285; georeferenceSources: GeoLocate; eventDate: 1968-06-10T00:00:00; startDayOfYear: 162; year: 1968; month: 6; day: 10; verbatimEventDate: 6/10/1968; individualCount: 1; preparations: pinned; catalogNumber: TTU-Z_219302; occurrenceDetails: http:// api.gbif.org/v1/occurrence/search?occurrenceld=6367d96d-1c14-488cbaf5-51c318d77b1a; recordedBy: D. D. Collins; otherCatalogNumbers: TTU1997-058; identifiedBy: C. W. O'Brien; dateldentified: 1978-01-01T00:00:00; modified: 2015-01-08T00:00:00.000+0000; rights: http://creativecommons.org/licenses/by-nc/3.0/; rightsHolder: Museum of Texas Tech University; accessRights: CC BY-NC (AttributionNon-Commercial); collectionID: d4e788b4-5d52-47a3-873e-227c6df49c7b; institutionCode: TTU; collectionCode: TTU-Z; basisOfRecord: PRESERVED_SPECIMEN; occurrencelD: 6367d96d-1c14-488c-baf5-51c318d77b1a

g. scientificName: Compsus auricephalus Say, 1824; taxonomicStatus: ACCEPTED; taxonID: 8801; kingdom: Animalia; phylum: Arthropoda; class: Insecta; order: Coleoptera; family: Curculionidae; taxonRank: SPECIES; genus: Compsus; specificEpithet: auricephalus; country: United States of America; countryCode: US; stateProvince: Texas; county: Hall; locality: Estelline; decimalLatitude: 34.54667; decimalLongitude: -100.43778; geodeticDatum: WGS84; coordinateUncertaintyInMeters: 1285; georeferenceSources: GeoLocate; eventDate: 1968-06-10T00:00:00; startDayOfYear: 162; year: 1968; month: 6; day: 10; verbatimEventDate: 6/10/1968; individualCount: 1; preparations: pinned; catalogNumber: TTU-Z_219303; occurrenceDetails: http:// api.gbif.org/v1/occurrence/search?occurrenceld=4712568f-cd4c-44b3-8709-e1f7f12ffd69; recordedBy: D. D. Collins; otherCatalogNumbers: TTU1997-058; identifiedBy: C.W. O'Brien; modified: 2015-01-08T00:00:00.000+0000; rights: http://creativecommons.org/ licenses/by-nc/3.0/; rightsHolder: Museum of Texas Tech University; accessRights: CC BY-NC (Attribution-Non-Commercial); collectionID: d4e788b4-5d52-47a3-873e-227c6df49c7b; institutionCode: TTU; collectionCode: TTU-Z; basisOfRecord: PRESERVED_SPECIMEN; occurrencelD: 4712568f-cd4c-44b3-8709e1f7f12ffd69

h. ScientificName: Compsus auricephalus Say, 1824; taxonomicStatus: ACCEPTED; taxonID: 8801; kingdom: Animalia; phylum: Arthropoda; class: Insecta; order: Coleoptera; family: Curculionidae; taxonRank: SPECIES; genus: Compsus; specificEpithet: auricephalus; country: United States of America; countryCode: US; stateProvince: Texas; county: Hall; locality: Estelline; decimalLatitude: 34.54667; decimalLongitude: -100.43778; geodeticDatum: WGS84; coordinateUncertaintyInMeters: 1285; georeferenceSources: GeoLocate; eventDate: 1968-06-10T00:00:00; startDayOfYear: 162; year: 1968; month: 6; day: 10; verbatimEventDate: 6/10/1968; individualCount: 1 ; preparations: pinned; catalogNumber: TTU-Z_219304; occurrenceDetails: http:// api.gbif.org/v1/occurrence/search?occurrenceld=90809cdc-23cb-426a-b5b9be2f6e0c7e09; recordedBy: D. D. Collins; otherCatalogNumbers: TTU1997-058; identifiedBy: C.W. O'Brien; dateldentified: 1970-01-01T00:00:00; modified: 2015-01-08T00:00:00.000+0000; rights: http://creativecommons.org/licenses/by-nc/3.0/; rightsHolder: Museum of Texas Tech University; accessRights: CC BY-NC (AttributionNon-Commercial); collectionID: d4e788b4-5d52-47a3-873e-227c6df49c7b; 
institutionCode: TTU; collectionCode: TTU-Z; basisOfRecord: PRESERVED_SPECIMEN; occurrencelD: 90809cdc-23cb-426a-b5b9-be2f6e0c7e09

i. scientificName: Compsus auricephalus Say, 1824; taxonomicStatus: ACCEPTED; taxonID: 8801; kingdom: Animalia; phylum: Arthropoda; class: Insecta; order: Coleoptera; family: Curculionidae; taxonRank: SPECIES; genus: Compsus; specificEpithet: auricephalus; country: United States of America; countryCode: US; stateProvince: Texas; county: Hall; locality: Estelline; decimalLatitude: 34.54667; decimalLongitude: -100.43778; geodeticDatum: WGS84; coordinateUncertaintyInMeters: 1285; georeferenceSources: GeoLocate; eventDate: 1968-06-10T00:00:00; startDayOfYear: 162; year: 1968; month: 6; day: 10; verbatimEventDate: 6/10/1968; individualCount: 1 ; preparations: pinned; catalogNumber: TTU-Z_219305; occurrenceDetails: http:// api.gbif.org/v1/occurrence/search? occurrenceld=a3d0f53f-5124-44c8-9e64-91f68b54daa6; recordedBy: D. D. Collins; otherCatalog Numbers: TTU1997-058; identifiedBy: C.W. O'Brien; dateldentified: 1970-01-01T00:00:00; modified: 2015-01-08T00:00:00.000+0000; rights: http:// creativecommons.org/licenses/by-nc/3.0/; rightsHolder: Museum of Texas Tech University; accessRights: CC BY-NC (Attribution-Non-Commercial); collectionID: d4e788b4-5d52-47a3-873e-227c6df49c7b; institutionCode: TTU; collectionCode: TTU-Z; basisOfRecord: PRESERVED_SPECIMEN; occurrenceID: a3d0f53f-5124-44c8-9e64-91f68b54daa6

j. ScientificName: Compsus auricephalus Say, 1824; taxonomicStatus: ACCEPTED; taxonID: 8801; kingdom: Animalia; phylum: Arthropoda; class: Insecta; order: Coleoptera; family: Curculionidae; taxonRank: SPECIES; genus: Compsus; specificEpithet: auricephalus; country: United States of America; countryCode: US; stateProvince: Texas; county: Hall; decimalLatitude: 34.54667 ; decimalLongitude: -100.43778 ; geodeticDatum: WGS84; coordinateUncertaintyInMeters: 1285; georeferenceSources: GeoLocate; eventDate: 1968-06-10T00:00:00; startDayOfYear: 162; year: 1968; month: 6; day: 10; verbatimEventDate: 6/10/1968; individualCount: 1; lifeStage: ADULT; preparations: pinned; catalogNumber: TTU-Z_219306; occurrenceDetails: http://api.gbif.org/v1/ occurrence/search?occurrenceld=a5bdd73f-4d99-4222-90e6-7290a1dda179; recordedBy: D. D. Collins; otherCatalogNumbers: TTU1997-058; identifiedBy: C.W. O'Brien; dateldentified: 1970-01-01T00:00:00; modified: 2015-01-08T00:00:00.000+0000; rights: http://creativecommons.org/licenses/by-nc/3.0/; rightsHolder: Museum of Texas Tech University; accessRights: CC BY-NC (Attribution-Non-Commercial); collectionID: d4e788b4-5d52-47a3-873e-227c6df49c7b; institutionCode: TTU; collectionCode: TTU-Z; basisOfRecord: PRESERVED_SPECIMEN; occurrenceID:

a5bdd73f-4d99-4222-90e6-7290a1dda179

k. scientificName: Compsus auricephalus Say, 1824; taxonomicStatus: ACCEPTED; taxonID: 8801; kingdom: Animalia; phylum: Arthropoda; class: Insecta; order: Coleoptera; family: Curculionidae; taxonRank: SPECIES; genus: Compsus; specificEpithet: auricephalus; country: United States of America; countryCode: US; stateProvince: Texas; county: Hall; locality: Estelline; decimalLatitude: 34.54667; decimalLongitude: -100.43778; geodeticDatum: WGS84; coordinateUncertaintylnMeters: 1285; georeferenceSources: GeoLocate; eventDate: 1968-06-10T00:00:00; startDayOfYear: 162; year: 1968; month: 6; day: 10; verbatimEventDate: 6/10/1968; individualCount: 1; preparations: pinned; catalogNumber: TTU-Z_219307; occurrenceDetails: http:// api.gbif.org/v1/occurrence/search?occurrenceld=b53e7fa2-baf4-4a03a3ca-769c3ded1023; recordedBy: D. D. Collins; otherCatalogNumbers: TTU1997-058; identifiedBy: C.W. O'Brien; dateldentified: 1970-01-01T00:00:00; modified: 2015-01-08T00:00:00.000+0000; rights: http://creativecommons.org/licenses/by-nc/3.0/; 
rightsHolder: Museum of Texas Tech University; accessRights: CC BY-NC (AttributionNon-Commercial); collectionID: d4e788b4-5d52-47a3-873e-227c6df49c7b; institutionCode: TTU; collectionCode: TTU-Z; basisOfRecord: PRESERVED_SPECIMEN; occurrencelD: b53e7fa2-baf4-4a03-a3ca-769c3ded1023

I. scientificName: Compsus auricephalus Say, 1824; taxonomicStatus: ACCEPTED; taxonID: 8801; kingdom: Animalia; phylum: Arthropoda; class: Insecta; order: Coleoptera; family: Curculionidae; taxonRank: SPECIES; genus: Compsus; specificEpithet: auricephalus; country: United States of America; countryCode: US; stateProvince: Texas; county: King; locality: Guthrie; decimalLatitude: 33.62056; decimalLongitude: -100.3225; geodeticDatum: WGS84; coordinateUncertaintylnMeters: 3036; georeferenceSources: GeoLocate; eventDate: 1968-06-18T00:00:00; startDayOfYear: 170; year: 1968; month: 6; day: 18; verbatimEventDate: 18-19 June 1968; individualCount: 1; preparations: pinned; catalogNumber: TTU-Z_219297; occurrenceDetails: http://api.gbif.org/v1/ occurrence/search?occurrenceld=308ccd72-428a-4119-aeb2-71cb9f4052e3; occurrenceRemarks: S - 108 - F; recordedBy: Wayne H. Swenson; otherCatalogNumbers: TTU1997-058; identifiedBy: R. E. Warner; modified: 2015-01-08T00:00:00.000+0000; rights: http://creativecommons.org/licenses/by-nc/3.0/; rightsHolder: Museum of Texas Tech University; accessRights: CC BY-NC (AttributionNon-Commercial); collectionID: d4e788b4-5d52-47a3-873e-227c6df49c7b; institutionCode: TTU; collectionCode: TTU-Z; basisOfRecord: PRESERVED_SPECIMEN; occurrencelD: 308ccd72-428a-4119-aeb2-71cb9f4052e3

m. scientificName: Compsus auricephalus Say, 1824; taxonomicStatus: ACCEPTED; taxonID: 8801; kingdom: Animalia; phylum: Arthropoda; class: Insecta; order: Coleoptera; family: Curculionidae; taxonRank: SPECIES; genus: Compsus; specificEpithet: auricephalus; country: United States of America; countryCode: US; stateProvince: Texas; county: King; locality: Guthrie; decimalLatitude: 33.62056; decimalLongitude: -100.3225 ; geodeticDatum: WGS84; coordinateUncertaintyInMeters: 3036; georeferenceSources: GeoLocate; eventDate: 1968-06-07T00:00:00; startDayOfYear: 159; year: 1968; month: 6; day: 7; verbatimEventDate: 7-Jun-68; individualCount: 1; preparations: pinned; catalogNumber: TTU-Z_219298; occurrenceDetails: http://api.gbif.org/v1/occurrence/ search?occurrenceld=42222edb-506a-413b-9b0f-ae59c8c38c93; recordedBy: Wayne H. Swenson; otherCatalogNumbers: TTU1997-058; identifiedBy: R. E. Warner; modified: 2014-07-25T00:00:00.000+0000; rights: http://creativecommons.org/licenses/by-nc/3.0/; rightsHolder: Museum of Texas Tech University; accessRights: CC BY-NC (AttributionNon-Commercial); collectionID: d4e788b4-5d52-47a3-873e-227c6df49c7b; institutionCode: TTU; collectionCode: TTU-Z; basisOfRecord: PRESERVED_SPECIMEN; occurrencelD: 42222edb-506a-413b-9b0f-ae59c8c38c93

n. ScientificName: Compsus auricephalus Say, 1824; taxonomicStatus: ACCEPTED; taxonID: 8801; kingdom: Animalia; phylum: Arthropoda; class: Insecta; order: Coleoptera; family: Curculionidae; taxonRank: SPECIES; genus: Compsus; specificEpithet: auricephalus; country: United States of America; countryCode: US; stateProvince: Texas; county: King; locality: Guthrie; decimalLatitude: 33.62056; decimalLongitude: -100.3225; geodeticDatum: WGS84; coordinateUncertaintyInMeters: 3036; georeferenceSources: GeoLocate; eventDate: 1968-06-18T00:00:00; startDayOfYear: 170; year: 1968; month: 6; day: 18; verbatimEventDate: 18-19 June 1968; individualCount: 1; preparations: pinned; catalogNumber: TTU-Z_219299; occurrenceDetails: http://api.gbif.org/v1/ occurrence/search?occurrenceld=cf7b5d95-e819-4092-84d0-355e0fbd132c; occurrenceRemarks: S - 108 - F; recordedBy: Wayne H. Swenson; otherCatalogNumbers: TTU1997-058; identifiedBy: R. E. Warner; modified: 2015-01-08T00:00:00.000+0000; rights: http://creativecommons.org/licenses/by-nc/3.0/; 
rightsHolder: Museum of Texas Tech University; accessRights: CC BY-NC (AttributionNon-Commercial); collectionID: d4e788b4-5d52-47a3-873e-227c6df49c7b; institutionCode: TTU; collectionCode: TTU-Z; basisOfRecord: PRESERVED_SPECIMEN; occurrencelD: cf7b5d95-e819-4092-84d0-355e0fbd132c

o. ScientificName: Compsus auricephalus Say, 1824; taxonomicStatus: ACCEPTED; taxonID: 8801; kingdom: Animalia; phylum: Arthropoda; class: Insecta; order: Coleoptera; family: Curculionidae; taxonRank: SPECIES; genus: Compsus; specificEpithet: auricephalus; country: United States of America; countryCode: US; stateProvince: Texas; county: King; locality: Guthrie; decimalLatitude: 33.62056; decimalLongitude: -100.3225; geodeticDatum: WGS84; coordinateUncertaintyInMeters: 3036; georeferenceSources: GeoLocate; eventDate: 1968-06-28T00:00:00; startDayOfYear: 180; year: 1968; month: 6; day: 28; verbatimEventDate: 28-Jun-68; individualCount: 1; preparations: pinned; catalogNumber: TTU-Z_219300; occurrenceDetails: http://api.gbif.org/v1/occurrence/ search?occurrenceld=b0f2136f-ac11-4e38-affd-cdb2e898bc06; occurrenceRemarks: S 108 - F; recordedBy: Wayne H. Swenson; otherCatalogNumbers: TTU1997-058; identifiedBy: R. E. Warner; modified: 2015-01-08T00:00:00.000+0000; rights: http:// creativecommons.org/licenses/by-nc/3.0/; rightsHolder: Museum of Texas Tech University; accessRights: CC BY-NC (Attribution-Non-Commercial); collectionID: d4e788b4-5d52-47a3-873e-227c6df49c7b; institutionCode: TTU; collectionCode: TTU-Z; basisOfRecord: PRESERVED_SPECIMEN; occurrencelD: b0f2136f-ac11-4e38-affdcdb2e898bc06

p. scientificName: Compsus auricephalus Say, 1824; taxonomicStatus: ACCEPTED; taxonID: 8801; kingdom: Animalia; phylum: Arthropoda; class: Insecta; order: Coleoptera; family: Curculionidae; taxonRank: SPECIES; genus: Compsus; specificEpithet: auricephalus; country: United States of America; countryCode: US; stateProvince: Texas; county: Motley; decimalLatitude: 34.074095; decimalLongitude: -100.779829; geodeticDatum: WGS84; coordinateUncertaintyInMeters: 43365; georeferenceRemarks: 89 High MOTLEY COUNTY; eventDate: 1968-06-25T00:00:00; startDayOfYear: 177; year: 1968; month: 6; day: 25; verbatimEventDate: 25-Jun-68; individualCount: 1; preparations: pinned; catalogNumber: TTU-Z_219021; occurrenceDetails: http:// api.gbif.org/v1/occurrence/search? occurrenceld=ecf5848d-2f28-437d-9c9a-314aa8859014; recordedBy: Robert Elkerson; otherCatalogNumbers: TTU1997-058; modified: 2015-01-08T00:00:00.000+0000; rights: http://creativecommons.org/licenses/by-nc/3.0/; rightsHolder: Museum of Texas Tech University; accessRights: CC BY-NC (Attribution-Non-Commercial); collectionID: d4e788b4-5d52-47a3-873e-227c6df49c7b; institutionCode: TTU; collectionCode: TTU-Z; basisOfRecord: PRESERVED_SPECIMEN; occurrenceID: ecf5848d-2f28-437d-9c9a-314aa8859014

q. scientificName: Compsus auricephalus Say, 1824; taxonomicStatus: ACCEPTED; taxonID: 8801; kingdom: Animalia; phylum: Arthropoda; class: Insecta; order: Coleoptera; family: Curculionidae; taxonRank: SPECIES; genus: Compsus; specificEpithet: auricephalus; country: United States of America; countryCode: US; stateProvince: Texas; county: Real; locality: Camp Wood; decimalLatitude: 29.66917; decimalLongitude: -100.01194; geodeticDatum: WGS84; coordinateUncertaintyInMeters: 952; georeferenceSources: GeoLocate; eventDate: 1968-06-01T00:00:00; startDayOfYear: 153; year: 1968; month: 6; day: 1; verbatimEventDate: 1 VI 1968; individualCount: 1 ; preparations: pinned; catalogNumber: TTU-Z_219022; occurrenceDetails: http:// api.gbif.org/v1/occurrence/search? occurrenceld=ea7be5d1-0592-4d4e-965b-9c7cf8674ed4; recordedBy: C. R. Ward; otherCatalogNumbers: TTU1997-058; modified: 2015-01-08T00:00:00.000+0000; rights: 
http://creativecommons.org/licenses/by-nc/3.0/; rightsHolder: Museum of Texas Tech University; accessRights: CC BY-NC (Attribution-Non-Commercial); collectionID: d4e788b4-5d52-47a3-873e-227c6df49c7b; institutionCode: TTU; collectionCode: TTU-Z; basisOfRecord: PRESERVED_SPECIMEN; occurrenceID: ea7be5d1-0592-4d4e-965b-9c7cf8674ed4

r. scientificName: Compsus auricephalus Say, 1824; taxonomicStatus: ACCEPTED; taxonID: 8801; kingdom: Animalia; phylum: Arthropoda; class: Insecta; order: Coleoptera; family: Curculionidae; taxonRank: SPECIES; genus: Compsus; specificEpithet: auricephalus; country: United States of America; countryCode: US; stateProvince: Texas; county: Jim Wells; locality: Alice; decimalLatitude: 27.75194; decimalLongitude: -98.06944; geodeticDatum: WGS84; coordinateUncertaintyInMeters: 6335; georeferenceSources: GeoLocate; eventDate: 1968-04-08T00:00:00; startDayOfYear: 99; year: 1968; month: 4; day: 8; verbatimEventDate: 4/8/1968; individualCount: 1 ; preparations: pinned; catalogNumber: TTU-Z_219301; occurrenceDetails: http:// api.gbif.org/v1/occurrence/search? occurrenceld=9a3e6e5a-4517-4a56-8b7f-3b98a0e6d3b9; recordedBy: R. R. Rogers; otherCatalogNumbers: TTU1997-058; modified: 2015-01-08T00:00:00.000+0000; rights: http://creativecommons.org/licenses/by-nc/3.0/; rightsHolder: Museum of Texas Tech University; accessRights: CC BY-NC (Attribution-Non-Commercial); collectionID: d4e788b4-5d52-47a3-873e-227c6df49c7b; institutionCode: TTU; collectionCode: TTU-Z; basisOfRecord: PRESERVED_SPECIMEN; occurrenceID:

9a3e6e5a-4517-4a56-8b7f-3b98a0e6d3b9

s. $\quad$ scientificName: Compsus auricephalus Say, 1824; taxonomicStatus: ACCEPTED; taxonID: 8801; kingdom: Animalia; phylum: Arthropoda; class: Insecta; order: Coleoptera; family: Curculionidae; taxonRank: SPECIES; genus: Compsus; specificEpithet: auricephalus; country: United States of America; countryCode: US; stateProvince: Texas; county: Mason; locality: $1 \mathrm{mi}$. south of Mason; decimalLatitude: 30.734094; decimalLongitude: -99.23028; geodeticDatum: WGS84; coordinateUncertaintyInMeters: 5405; georeferenceSources: GeoLocate; eventDate: 1970-10-01T00:00:00; startDayOfYear: 274; endDayOfYear: -30; year: 1970; month: 10; day: 1; verbatimEventDate: $\mathrm{X}-1$ - 70; individualCount: 1; preparations: pinned; catalogNumber: TTU-Z_219308; occurrenceDetails: http://api.gbif.org/v1/occurrence/search? occurrenceld=f20cea5b-2318-465b-aaa0-947e63f12e64; recordedBy: J. A. Campbell; otherCatalogNumbers: TTU1997-058; modified: 2015-01-08T00:00:00.000+0000; rights: http://creativecommons.org/licenses/by-nc/3.0/; rightsHolder: Museum of Texas Tech University; accessRights: CC BY-NC (Attribution-Non-Commercial); collectionID: d4e788b4-5d52-47a3-873e-227c6df49c7b; institutionCode: TTU; collectionCode: TTU-Z; basisOfRecord: PRESERVED_SPECIMEN; occurrenceID: f20cea5b-2318-465baaa0-947e63f12e64

t. ScientificName: Compsus auricephalus Say, 1824; taxonomicStatus: ACCEPTED; taxonID: 8801; kingdom: Animalia; phylum: Arthropoda; class: Insecta; order: Coleoptera; family: Curculionidae; taxonRank: SPECIES; genus: Compsus; specificEpithet: auricephalus; country: United States of America; countryCode: US; stateProvince: Texas; county: San Patricio; locality: Welder Wildlife Refuge; verbatimElevation: 800'; decimalLatitude: 28.122069; decimalLongitude: -97.442532; geodeticDatum: WGS84; coordinateUncertaintyInMeters: 100; georeferencedBy: alexa.davis (2014-07-31 14:47:25); georeferenceSources: georef batch tool 2014-07-31; GeoLocate; georeferenceVerificationStatus: reviewed - high confidence; eventDate: 1971-04-02T00:00:00; startDayOfYear: 92; year: 1971; month: 4; day: 2; verbatimEventDate: IV-2-1971; individualCount: 1; lifeStage: ADULT; preparations: 
pinned; catalogNumber: TTU-Z_219310; occurrenceDetails: http://api.gbif.org/v1/ occurrence/search?occurrenceld=483419bc-e5e1-4478-aace-5a5394fdd918; occurrenceRemarks: mesquite Project Texas Tech University; recordNumber: 219310; recordedBy: L. B. O'Brien; otherCatalogNumbers: TTU1997-058; identifiedBy: C. W. O'Brien; dateldentified: 1972-01-01T00:00:00; modified: 2014-07-31T00:00:00.000+0000; rights: http://creativecommons.org/licenses/by-nc/3.0/; rightsHolder: Museum of Texas Tech University; accessRights: CC BY-NC (Attribution-Non-Commercial); collectionID: d4e788b4-5d52-47a3-873e-227c6df49c7b; institutionCode: TTU; collectionCode: TTU-Z; basisOfRecord: PRESERVED_SPECIMEN; occurrenceID: 483419bc-e5e1-4478aace-5a5394fdd918

u. scientificName: Compsus auricephalus Say, 1824; taxonomicStatus: ACCEPTED; taxonID: 8801; kingdom: Animalia; phylum: Arthropoda; class: Insecta; order: Coleoptera; family: Curculionidae; taxonRank: SPECIES; genus: Compsus; specificEpithet: auricephalus; country: United States of America; countryCode: US; stateProvince: Texas; county: Jim Wells; decimalLatitude: 27.731338; decimalLongitude: -98.089886; geodeticDatum: WGS84; coordinateUncertaintyInMeters: 57990; georeferenceRemarks: 92 High JIM WELLS COUNTY; eventDate: 1969-04-03T00:00:00; startDayOfYear: 93; year: 1969; month: 4; day: 3; verbatimEventDate: 3-Apr-69; individualCount: 1; preparations: pinned; catalogNumber: TTU-Z_219023; occurrenceDetails: http:// api.gbif.org/v1/occurrence/search?occurrenceld=1314bdaacf31-47ce-9102-9f4659b8c7d4; recordedBy: K. Kattner; otherCatalogNumbers: TTU1997-058; modified: 2015-01-08T00:00:00.000+0000; rights: http:// creativecommons.org/licenses/by-nc/3.0/; rightsHolder: Museum of Texas Tech University; accessRights: CC BY-NC (Attribution-Non-Commercial); collectionID: d4e788b4-5d52-47a3-873e-227c6df49c7b; institutionCode: TTU; collectionCode: TTU-Z; basisOfRecord: PRESERVED_SPECIMEN; occurrenceID: 1314bdaacf31-47ce-9102-9f4659b8c7d4

v. ScientificName: Compsus auricephalus Say, 1824; taxonomicStatus: ACCEPTED; taxonID: 8801; kingdom: Animalia; phylum: Arthropoda; class: Insecta; order: Coleoptera; family: Curculionidae; taxonRank: SPECIES; genus: Compsus; specificEpithet: auricephalus; country: United States of America; countryCode: US; stateProvince: Texas; county: Garza; locality: Road side Park on Caprock just NE of Post; decimalLatitude: 33.19083; decimalLongitude: -101.37778; geodeticDatum: WGS84; coordinateUncertaintyInMeters: 2337; georeferencedBy: Alex Gregg (2014-07-30 12:00:49); georeferenceSources: georef batch tool 2014-07-30; GeoLocate; georeferenceVerificationStatus: reviewed - high confidence; eventDate: 2007-05-16T00:00:00; startDayOfYear: 136; year: 2007; month: 5; day: 16; verbatimEventDate: 16-V-2007; individualCount: 1; lifeStage: ADULT; preparations: dry pinned; catalogNumber: TTU-Z_050014; occurrenceDetails: http://api.gbif.org/v1/ occurrence/search?occurrenceld=5501285b-d654-4b55-b188-6155bd373f85; occurrenceRemarks: On Mesquite Tree; recordedBy: James C. Cokendolpher; J.Creel; otherCatalogNumbers: TTU1997-058; identifiedBy: R. S. Anderson; dateldentified: 2017-01-01T00:00:00; modified: 2015-01-08T00:00:00.000+0000; rights: http:// creativecommons.org/licenses/by-nc/3.0/; rightsHolder: Museum of Texas Tech University; accessRights: CC BY-NC (Attribution-Non-Commercial); collectionID: d4e788b4-5d52-47a3-873e-227c6df49c7b; institutionCode: TTU; collectionCode: TTU-Z; basisOfRecord: PRESERVED_SPECIMEN; occurrenceID: 5501285b-d654-4b55b188-6155bd373f85

w. scientificName: Compsus auricephalus Say, 1824; taxonomicStatus: ACCEPTED; taxonID: 8801; kingdom: Animalia; phylum: Arthropoda; class: Insecta; order: Coleoptera; 
family: Curculionidae; taxonRank: SPECIES; genus: Compsus; specificEpithet: auricephalus; country: United States of America; countryCode: US; stateProvince: Texas; county: Dickens; locality: 7-Bar Ranch; decimalLatitude: 33.758961; decimalLongitude: -100.755789; geodeticDatum: WGS84; coordinateUncertaintyInMeters: 100; georeferencedBy: luis.tirado (2014-08-01 20:13:56); georeferenceSources: georef batch tool 2014-08-01; GeoLocate; georeferenceVerificationStatus: reviewed - high confidence; eventDate: 1972-05-17T00:00:00; startDayOfYear: 138; year: 1972; month: 5; day: 17; verbatimEventDate: 5/17/1972; individualCount: 1; lifeStage: ADULT; preparations: pinned; catalogNumber: TTU-Z_219288; occurrenceDetails: http://api.gbif.org/v1/ occurrence/search?occurrenceld=bed89b36-3a63-405b-879e-bf85666ded92;

recordedBy: L. B. Smith; otherCatalogNumbers: TTU1997-058; identifiedBy: L. B. Smith; modified: 2015-01-08T00:00:00.000+0000; rights: http://creativecommons.org/licenses/ by-nc/3.0/; rightsHolder: Museum of Texas Tech University; accessRights: CC BY-NC (Attribution-Non-Commercial); collectionID: d4e788b4-5d52-47a3-873e-227c6df49c7b; institutionCode: TTU; collectionCode: TTU-Z; basisOfRecord: PRESERVED_SPECIMEN; occurrencelD: bed89b36-3a63-405b-879e-bf85666ded92

x. scientificName: Compsus auricephalus Say, 1824; taxonomicStatus: ACCEPTED; taxonID: 8801; kingdom: Animalia; phylum: Arthropoda; class: Insecta; order: Coleoptera; family: Curculionidae; taxonRank: SPECIES; genus: Compsus; specificEpithet: auricephalus; country: United States of America; countryCode: US; stateProvince: Texas; county: Dickens; locality: 7-Bar Ranch; decimalLatitude: 33.758961; decimalLongitude: -100.755789; geodeticDatum: WGS84; coordinateUncertaintyInMeters: 100; georeferencedBy: luis.tirado (2014-08-01 20:13:56); georeferenceSources: georef batch tool 2014-08-01; GeoLocate; georeferenceVerificationStatus: reviewed - high confidence; eventDate: 1972-05-17T00:00:00; startDayOfYear: 138; year: 1972; month: 5; day: 17; verbatimEventDate: 5/17/1972; individualCount: 1; lifeStage: ADULT; preparations: pinned; catalogNumber: TTU-Z_219287; occurrenceDetails: http://api.gbif.org/v1/ occurrence/search?occurrenceld=78a280bd-fead-404d-afba-9ab04e1ff1ce; recordedBy: L. B. Smith; otherCatalogNumbers: TTU1997-058; identifiedBy: L. B. Smith; modified: 2015-01-08T00:00:00.000+0000; rights: http://creativecommons.org/licenses/by-nc/3.0/; rightsHolder: Museum of Texas Tech University; accessRights: CC BY-NC (AttributionNon-Commercial); collectionID: d4e788b4-5d52-47a3-873e-227c6df49c7b; institutionCode: TTU; collectionCode: TTU-Z; basisOfRecord: PRESERVED_SPECIMEN; occurrencelD: 78a280bd-fead-404d-afba-9ab04e1ff1ce

y. $\quad$ scientificName: Compsus auricephalus Say, 1824; taxonomicStatus: ACCEPTED; taxonID: 8801; kingdom: Animalia; phylum: Arthropoda; class: Insecta; order: Coleoptera; family: Curculionidae; taxonRank: SPECIES; genus: Compsus; specificEpithet: auricephalus; country: United States of America; countryCode: US; stateProvince: Mississippi; county: Warren; decimalLatitude: 32.35723; decimalLongitude: -90.852011; geodeticDatum: WGS84; coordinateUncertaintyInMeters: 43674; georeferenceRemarks: 89 High WARREN COUNTY; eventDate: 1973-06-01T00:00:00; startDayOfYear: 152; year: 1973; month: 6; day: 1; verbatimEventDate: 1-Jun-73; individualCount: 1; preparations: pinned; catalogNumber: TTU-Z_219295; occurrenceDetails: http:// api.gbif.org/v1/occurrence/search?occurrenceld=8bd7da64-4d84-4260b606-34cef413c301; recordedBy: Bryant Mather; otherCatalogNumbers: TTU1997-058; identifiedBy: C.W. O'Brien; dateldentified: 1974-01-01T00:00:00; modified: 2015-01-08T00:00:00.000+0000; rights: http://creativecommons.org/licenses/by-nc/3.0/; rightsHolder: Museum of Texas Tech University; accessRights: CC BY-NC (AttributionNon-Commercial); collectionID: d4e788b4-5d52-47a3-873e-227c6df49c7b; 
institutionCode: TTU; collectionCode: TTU-Z; basisOfRecord: PRESERVED_SPECIMEN; occurrencelD: 8bd7da64-4d84-4260-b606-34cef413c301

z. ScientificName: Compsus auricephalus Say, 1824; taxonomicStatus: ACCEPTED; taxonID: 8801; kingdom: Animalia; phylum: Arthropoda; class: Insecta; order: Coleoptera; family: Curculionidae; taxonRank: SPECIES; genus: Compsus; specificEpithet: auricephalus; country: United States of America; countryCode: US; stateProvince: Mississippi; county: Warren; decimalLatitude: 32.35723; decimalLongitude: -90.852011; geodeticDatum: WGS84; coordinateUncertaintyInMeters: 43674; georeferenceRemarks: 89 High WARREN COUNTY; eventDate: 1973-06-18T00:00:00; startDayOfYear: 169; year: 1973; month: 6; day: 18; verbatimEventDate: 18-Jun-73; individualCount: 1; preparations: pinned; catalogNumber: TTU-Z_219296; occurrenceDetails: http:// api.gbif.org/v1/occurrence/search? occurrenceld=6d0aa9ae-9f7e-4226-952d-47ebf9bfdc71; recordedBy: Bryant Mather; otherCatalog Numbers: TTU1997-058; identifiedBy: C.W. O'Brien; dateldentified: 1974-01-01T00:00:00; modified: 2015-01-08T00:00:00.000+0000; rights: http:// creativecommons.org/licenses/by-nc/3.0/; rightsHolder: Museum of Texas Tech University; accessRights: CC BY-NC (Attribution-Non-Commercial); collectionID: d4e788b4-5d52-47a3-873e-227c6df49c7b; institutionCode: TTU; collectionCode: TTU-Z; basisOfRecord: PRESERVED_SPECIMEN; occurrenceID:

6d0aa9ae-9f7e-4226-952d-47ebf9bfdc71

aa. ScientificName: Compsus auricephalus Say, 1824; taxonomicStatus: ACCEPTED; taxonID: 8801; kingdom: Animalia; phylum: Arthropoda; class: Insecta; order: Coleoptera; family: Curculionidae; taxonRank: SPECIES; genus: Compsus; specificEpithet: auricephalus; country: United States of America; countryCode: US; stateProvince: Texas; county: Cameron; locality: Palmetto Hill, 10 m w Boca Chica; decimalLatitude: 25.977545; decimalLongitude: -97.351891; geodeticDatum: WGS84; coordinateUncertaintyInMeters: 500; georeferencedBy: luis.tirado (2014-08-01 20:01:53); georeferenceSources: georef batch tool 2014-08-01; GeoLocate; georeferenceVerificationStatus: reviewed - high confidence; eventDate: 1985-10-13T00:00:00; startDayOfYear: 286; year: 1985; month: 10; day: 13; verbatimEventDate: Oct. 13 1985; individualCount: 1; preparations: pinned; catalogNumber: TTU-Z_219289; occurrenceDetails: http://api.gbif.org/v1/occurrence/ search?occurrenceld=b679d423-1867-4788-ac0f-45c56fbf0ded; recordedBy: R. Morris II.; otherCatalogNumbers: TTU1997-058; identifiedBy: Downie; modified: 2015-01-08T00:00:00.000+0000; rights: http://creativecommons.org/licenses/by-nc/3.0/; rightsHolder: Museum of Texas Tech University; accessRights: CC BY-NC (AttributionNon-Commercial); collectionID: d4e788b4-5d52-47a3-873e-227c6df49c7b; institutionCode: TTU; collectionCode: TTU-Z; basisOfRecord: PRESERVED_SPECIMEN; occurrencelD: b679d423-1867-4788-ac0f-45c56fbf0ded

ab. ScientificName: Compsus auricephalus Say, 1824; taxonomicStatus: ACCEPTED; taxonID: 8801; kingdom: Animalia; phylum: Arthropoda; class: Insecta; order: Coleoptera; family: Curculionidae; taxonRank: SPECIES; genus: Compsus; specificEpithet: auricephalus; country: United States of America; countryCode: US; stateProvince: Texas; county: Cameron; locality: Palmetto Hill, 10 m w Boca Chica; decimalLatitude: 25.977545; decimalLongitude: -97.351891; geodeticDatum: WGS84; coordinateUncertaintyInMeters: 500; georeferencedBy: luis.tirado (2014-08-01 20:01:53); georeferenceSources: georef batch tool 2014-08-01; GeoLocate; georeferenceVerificationStatus: reviewed - high confidence; eventDate: 1985-10-13T00:00:00; startDayOfYear: 286; year: 1985; month: 10; day: 13; verbatimEventDate: Oct. 13 1985; individualCount: 1; preparations: pinned; catalogNumber: TTU-Z_219290; occurrenceDetails: http://api.gbif.org/v1/occurrence/ search?occurrenceld=8e647254-b380-4d0c-957f-b0ac420bd757; recordedBy: R. Morris 
II.; otherCatalog Numbers: TTU1997-058; identifiedBy: C.W. O'Brien; dateldentified: 1974-01-01T00:00:00; modified: 2015-01-08T00:00:00.000+0000; rights: http:// creativecommons.org/licenses/by-nc/3.0/; rightsHolder: Museum of Texas Tech University; accessRights: CC BY-NC (Attribution-Non-Commercial); collectionID: d4e788b4-5d52-47a3-873e-227c6df49c7b; institutionCode: TTU; collectionCode: TTU-Z; basisOfRecord: PRESERVED_SPECIMEN; occurrenceID: 8e647254-b380-4d0c-957fb0ac420bd757

ac. scientificName: Compsus auricephalus Say, 1824; taxonomicStatus: ACCEPTED; taxonID: 8801; kingdom: Animalia; phylum: Arthropoda; class: Insecta; order: Coleoptera; family: Curculionidae; taxonRank: SPECIES; genus: Compsus; specificEpithet: auricephalus; country: United States of America; countryCode: US; stateProvince: Texas; county: Cameron; locality: Palmetto Hill, $10 \mathrm{~m}$ w Boca Chica; decimalLatitude: 25.977545; decimalLongitude: -97.351891; geodeticDatum: WGS84; coordinateUncertaintyInMeters: 500; georeferencedBy: luis.tirado (2014-08-01 20:01:53); georeferenceSources: georef batch tool 2014-08-01; GeoLocate; georeferenceVerificationStatus: reviewed - high confidence; eventDate: 1985-10-13T00:00:00; startDayOfYear: 286; year: 1985; month: 10; day: 13; verbatimEventDate: Oct. 13 1985; individualCount: 1; preparations: pinned; catalogNumber: TTU-Z_219291; occurrenceDetails: http://api.gbif.org/v1/occurrence/ search?occurrenceld=b15e19a4-a01e-4566-9c18-bbe083e0ecc0; recordedBy: R. Morris II.; otherCatalogNumbers: TTU1997-058; identifiedBy: C.W. O'Brien; dateldentified: 1974-01-01T00:00:00; modified: 2015-01-08T00:00:00.000+0000; rights: http:// creativecommons.org/licenses/by-nc/3.0/; rightsHolder: Museum of Texas Tech University; accessRights: CC BY-NC (Attribution-Non-Commercial); collectionID: d4e788b4-5d52-47a3-873e-227c6df49c7b; institutionCode: TTU; collectionCode: TTU-Z; basisOfRecord: PRESERVED_SPECIMEN; occurrenceID: b15e19a4-a01e-4566-9c18bbe083e0ecc0

ad. ScientificName: Compsus auricephalus Say, 1824; taxonomicStatus: ACCEPTED; taxonID: 8801; kingdom: Animalia; phylum: Arthropoda; class: Insecta; order: Coleoptera; family: Curculionidae; taxonRank: SPECIES; genus: Compsus; specificEpithet: auricephalus; country: United States of America; countryCode: US; stateProvince: Texas; county: Cameron; locality: Palmetto Hill, 10 m w Boca Chica; decimalLatitude: 25.977545; decimalLongitude: -97.351891; geodeticDatum: WGS84; coordinateUncertaintyInMeters: 500; georeferencedBy: luis.tirado (2014-08-01 20:01:53); georeferenceSources: georef batch tool 2014-08-01; GeoLocate; georeferenceVerificationStatus: reviewed - high confidence; eventDate: 1985-10-13T00:00:00; startDayOfYear: 286; year: 1985; month: 10; day: 13; verbatimEventDate: Oct. 13 1985; individualCount: 1; preparations: pinned; catalogNumber: TTU-Z_219292; occurrenceDetails: http://api.gbif.org/v1/occurrence/ search?occurrenceld=b534485d-87b2-45d8-8fd7-ef96f9d0e34d; recordedBy: R. Morris II.; otherCatalogNumbers: TTU1997-058; identifiedBy: C.W. O'Brien; dateldentified: 1974-01-01T00:00:00; modified: 2015-01-08T00:00:00.000+0000; rights: http:// creativecommons.org/licenses/by-nc/3.0/; rightsHolder: Museum of Texas Tech University; accessRights: CC BY-NC (Attribution-Non-Commercial); collectionID: d4e788b4-5d52-47a3-873e-227c6df49c7b; institutionCode: TTU; collectionCode: TTU-Z; basisOfRecord: PRESERVED_SPECIMEN; occurrenceID: b534485d-87b2-45d8-8fd7ef96f9d0e34d

ae. $\quad$ scientificName: Compsus auricephalus Say, 1824; taxonomicStatus: ACCEPTED; taxonID: 8801; kingdom: Animalia; phylum: Arthropoda; class: Insecta; order: Coleoptera; family: Curculionidae; taxonRank: SPECIES; genus: Compsus; specificEpithet: auricephalus; country: United States of America; countryCode: US; stateProvince: Texas; county: Cameron; locality: Palmetto Hill, 10 m w Boca Chica; decimalLatitude: 25.977545; 
decimalLongitude: -97.351891; geodeticDatum: WGS84; coordinateUncertaintyInMeters: 500; georeferencedBy: luis.tirado (2014-08-01 20:01:53); georeferenceSources: georef batch tool 2014-08-01; GeoLocate; georeferenceVerificationStatus: reviewed - high confidence; eventDate: 1985-10-13T00:00:00; startDayOfYear: 286; year: 1985; month: 10; day: 13; verbatimEventDate: Oct. 13 1985; individualCount: 1; preparations: pinned; catalogNumber: TTU-Z_219293; occurrenceDetails: http://api.gbif.org/v1/occurrence/ search?occurrenceld=92e14681-3a2f-4a7d-80a2-1 bade6908523; recordedBy: R. Morris II.; otherCatalogNumbers: TTU1997-058; identifiedBy: C.W. O'Brien; dateldentified: 1974-01-01T00:00:00; modified: 2015-01-08T00:00:00.000+0000; rights: http:// creativecommons.org/licenses/by-nc/3.0/; rightsHolder: Museum of Texas Tech University; accessRights: CC BY-NC (Attribution-Non-Commercial); collectionID: d4e788b4-5d52-47a3-873e-227c6df49c7b; institutionCode: TTU; collectionCode: TTU-Z; basisOfRecord: PRESERVED_SPECIMEN; occurrenceID:

92e14681-3a2f-4a7d-80a2-1bade6908523

af. ScientificName: Compsus auricephalus Say, 1824; taxonomicStatus: ACCEPTED; taxonID: 8801; kingdom: Animalia; phylum: Arthropoda; class: Insecta; order: Coleoptera; family: Curculionidae; taxonRank: SPECIES; genus: Compsus; specificEpithet: auricephalus; country: United States of America; countryCode: US; stateProvince: Texas; county: Cameron; locality: Palmetto Hill, 10 m w Boca Chica; decimalLatitude: 25.977545; decimalLongitude: -97.351891; geodeticDatum: WGS84; coordinateUncertaintyInMeters: 500; georeferencedBy: luis.tirado (2014-08-01 20:01:53); georeferenceSources: georef batch tool 2014-08-01; GeoLocate; georeferenceVerificationStatus: reviewed - high confidence; eventDate: 1985-10-13T00:00:00; startDayOfYear: 286; year: 1985; month: 10; day: 13; verbatimEventDate: Oct. 13 1985; individualCount: 1; preparations: pinned; catalogNumber: TTU-Z_219294; occurrenceDetails: http://api.gbif.org/v1/occurrence/ search?occurrenceld=0a6c95e9-5f56-4c10-9cd7-a116973b7bb5; recordedBy: R. Morris II.; otherCatalogNumbers: TTU1997-058; identifiedBy: C.W. O'Brien; dateldentified: 1974-01-01T00:00:00; modified: 2015-01-08T00:00:00.000+0000; rights: http:// creativecommons.org/licenses/by-nc/3.0/; rightsHolder: Museum of Texas Tech University; accessRights: CC BY-NC (Attribution-Non-Commercial); collectionID: d4e788b4-5d52-47a3-873e-227c6df49c7b; institutionCode: TTU; collectionCode: TTU-Z; basisOfRecord: PRESERVED_SPECIMEN; occurrenceID: 0a6c95e9-5f56-4c10-9cd7a116973b7bb5

ag. $\quad$ scientificName: Compsus auricephalus Say, 1824; taxonomicStatus: ACCEPTED; taxonID: 8801; kingdom: Animalia; phylum: Arthropoda; class: Insecta; order: Coleoptera; family: Curculionidae; taxonRank: SPECIES; genus: Compsus; specificEpithet: auricephalus; country: United States of America; countryCode: US; stateProvince: Texas; county: Lubbock; decimalLatitude: 33.610206; decimalLongitude: -101.820546; geodeticDatum: WGS84; coordinateUncertaintyInMeters: 41227; georeferenceRemarks: 90 High LUBBOCK COUNTY; eventDate: 1951-04-11T00:00:00; startDayOfYear: 101; year: 1951; month: 4; day: 11; verbatimEventDate: 4/11/1951; individualCount: 1; lifeStage: ADULT; preparations: dry pinned; catalogNumber: TTU-Z_224142; occurrenceDetails: http://api.gbif.org/v1/occurrence/search? occurrenceld=a46cfd85-0763-4003-9e75-e23cb53a20cc; recordedBy: J. Hatchett; otherCatalogNumbers: TTU1997-058; modified: 2015-01-08T00:00:00.000+0000; rights: http://creativecommons.org/licenses/by-nc/3.0/; rightsHolder: Museum of Texas Tech University; accessRights: CC BY-NC (Attribution-Non-Commercial); collectionID: d4e788b4-5d52-47a3-873e-227c6df49c7b; institutionCode: TTU; collectionCode: TTU-Z; basisOfRecord: PRESERVED_SPECIMEN; occurrenceID: a46cfd85-0763-4003-9e75e23cb53a20cc 
ah. scientificName: Compsus auricephalus Say, 1824; taxonomicStatus: ACCEPTED; taxonID: 8801; kingdom: Animalia; phylum: Arthropoda; class: Insecta; order: Coleoptera; family: Curculionidae; taxonRank: SPECIES; genus: Compsus; specificEpithet: auricephalus; country: United States of America; countryCode: US; stateProvince: Texas; county: San Patricio; locality: Welder Wildlife Refuge, Pollita Lake; decimalLatitude: 28.11228; decimalLongitude: -97.41609; geodeticDatum: WGS84; coordinateUncertaintyInMeters: 500; georeferencedBy: Alex Gregg (2014-08-07 14:37:43); georeferenceSources: georef batch tool 2014-08-07; GeoLocate; georeferenceVerificationStatus: reviewed - high confidence; samplingProtocol: at night; eventDate: 1971-04-03T00:00:00; startDayOfYear: 93; year: 1971; month: 4; day: 3; verbatimEventDate: IV-3-1971; individualCount: 1; lifeStage: ADULT; preparations: Pointed; catalogNumber: TTU-Z_223955; occurrenceDetails: http://api.gbif.org/v1/ occurrence/search?occurrenceld=d53c30d3-5f8f-4079-b90c-8c40b72a14e2; otherCatalogNumbers: TTU1997-058; identifiedBy: J. Girón; dateldentified: 2020-01-01T00:00:00; modified: 2015-01-08T00:00:00.000+0000; rights: http:// creativecommons.org/licenses/by-nc/3.0/; rightsHolder: Museum of Texas Tech University; accessRights: CC BY-NC (Attribution-Non-Commercial); collectionID: d4e788b4-5d52-47a3-873e-227c6df49c7b; institutionCode: TTU; collectionCode: TTU-Z; basisOfRecord: PRESERVED_SPECIMEN; occurrencelD: d53c30d3-5f8f-4079b90c-8c40b72a14e2

ai. ScientificName: Compsus auricephalus Say, 1824; taxonomicStatus: ACCEPTED; taxonID: 8801; kingdom: Animalia; phylum: Arthropoda; class: Insecta; order: Coleoptera; family: Curculionidae; taxonRank: SPECIES; genus: Compsus; specificEpithet: auricephalus; country: United States of America; countryCode: US; stateProvince: Texas; county: Garza; decimalLatitude: 33.179876; decimalLongitude: -101.298424; geodeticDatum: WGS84; coordinateUncertaintyInMeters: 41220; georeferenceRemarks: 88 High GARZA COUNTY; eventDate: 1956-04-21T00:00:00; startDayOfYear: 112; year: 1956; month: 4; day: 21; verbatimEventDate: 4/21/1956; individualCount: 1 ; lifeStage: ADULT; preparations: Pointed; catalogNumber: TTU-Z_224887; occurrenceDetails: http:// api.gbif.org/v1/occurrence/search?occurrenceld=c3ff87d1-25d3-4756af25-3085e50fbe4b; recordedBy: J. Boren; otherCatalogNumbers: TTU1997-058; modified: 2015-01-08T00:00:00.000+0000; rights: http://creativecommons.org/licenses/ by-nc/3.0/; rightsHolder: Museum of Texas Tech University; accessRights: CC BY-NC (Attribution-Non-Commercial); collectionID: d4e788b4-5d52-47a3-873e-227c6df49c7b; institutionCode: TTU; collectionCode: TTU-Z; basisOfRecord: PRESERVED_SPECIMEN; occurrencelD: c3ff87d1-25d3-4756-af25-3085e50fbe4b

\section{Description}

Body length 8-12 mm, width 3-4 mm; shape oval, length/width ratio 2.4-2.6; greatest width near mid-length of elytra in males, near posterior third in females. Integument dark brown to black; coverage composed of densely and evenly arranged, overlapping scales (Figs 1, 3A-C), mostly to completely covering integument, subcircular to distally angulate, appressed; scales uniformly pearly white to light brown (Fig. 1C, Fig. 3A-C) or iridescent light green along body with iridescent pink along dorsal surface of head and along anterior and ventral surfaces of legs (Fig. 1A, B, Fig. 3F); seta-like narrow scales sparsely and evenly arranged, relatively short and thick, recurvate, pale white to translucent. 

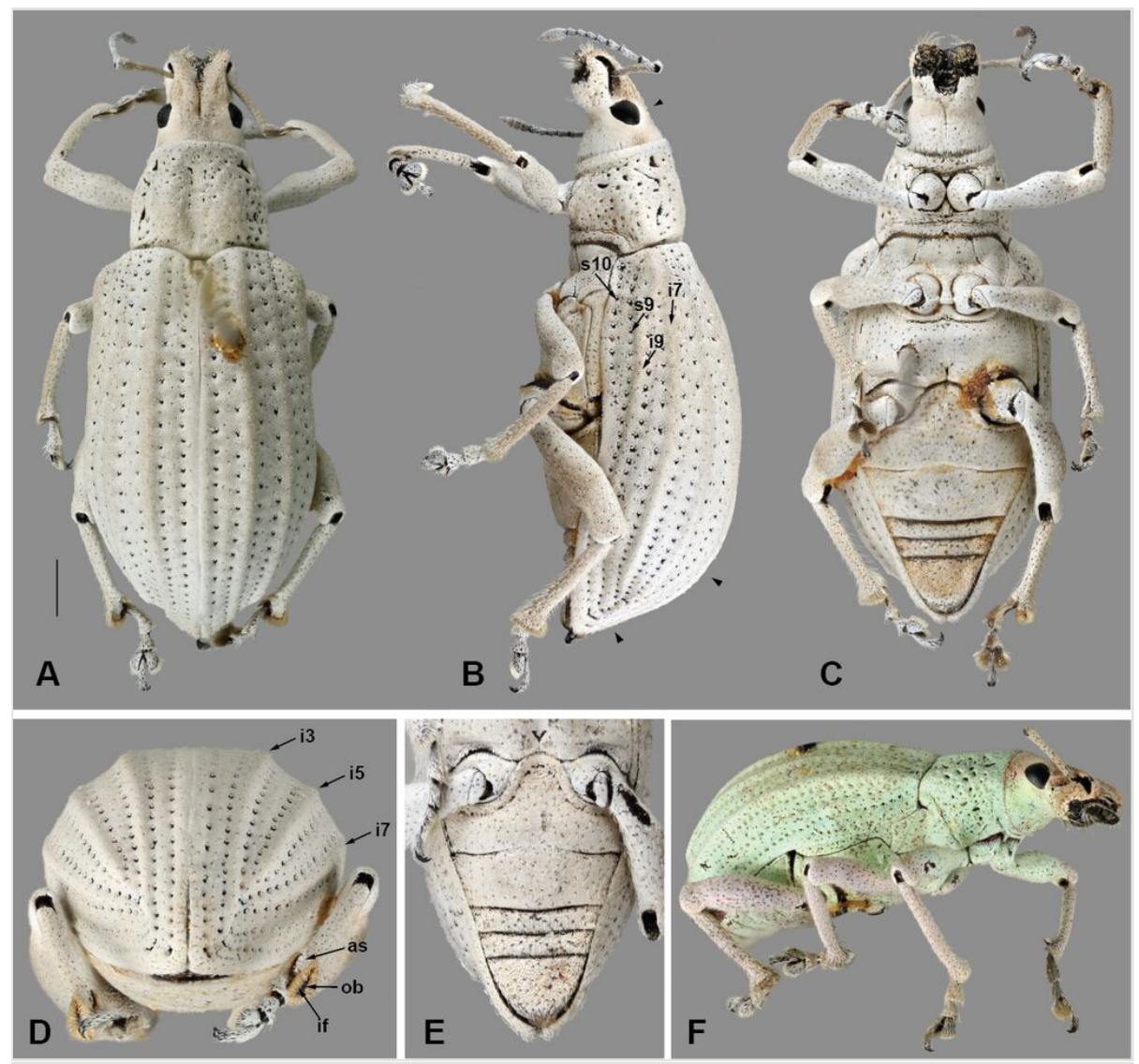

Figure 3. doi

Morphological features of Compsus auricephalus: (A-D) Texas, Garza Co. (TTU-Z_050014); scale bar: $1 \mathrm{~mm}$ : $(A)$ dorsal habitus, $(B)$ lateral habitus (s10: stria 10; s9: stria 9; i7: interstria 7; i9: interstria 9), (C) ventral habitus of female, (D) posterior view (i3: interstria 3; i5: interstria 5; i7: interstria 7; as: articular surface; ob: outer bevel; if: inner fringe), (E) Texas, Cameron Co. (TTU-Z_219291) ventral habitus of male; (F) Mississippi, Warren Co. (TTU-Z_219296), anterolateral habitus.

Head. Frons nearly flat, only very slightly transversally impressed at level of posterior margin of eyes; frons with deep, large median fovea (Fig. 4A, C, mf); surface of head densely covered by overlapping scales; scales apically angulate along base of head, progressing to oval anteriorly (Fig. 4B); curved, thick setae only along dorsal surface of head. Eyes in lateral view (Fig. 4B) tear-drop shaped, 1.5-times longer than wide, with acute margin pointing antero-ventrally; eyes mostly lateral, separated from anterolateral margin of prothorax by distance slightly shorter than greatest width of eye; in dorsal view (Fig. 4A, E-G), eyes moderately convex, with inner margins slightly oblique, interocular distance 4.6-times maximum width of eye. 

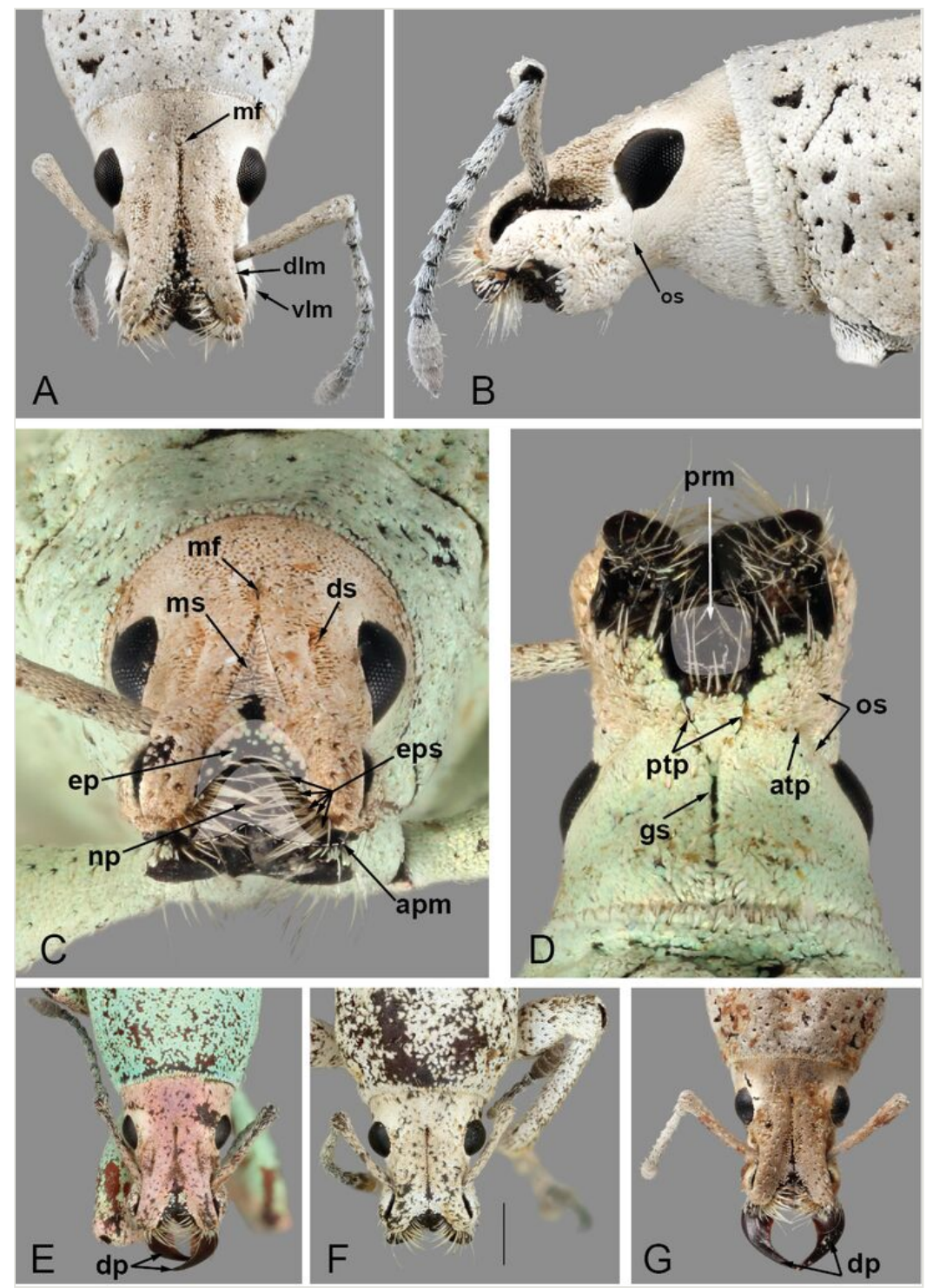

Figure 4. doi

Morphological features of the head of Compsus auricephalus: (A-B) Texas, Garza Co. (TTUZ_050014): (A) dorsal view (mf: median fovea; dlm: dorsolateral margin; vlm: ventrolateral margin), (B) lateral view (os: occipital suture); (C-D) Mississippi, Warren Co. (TTU-Z_219296): (C) head, anterior view (mf: median fovea; ms: median sulcus; ds: dorsolateral sulcus; ep: epistoma; eps: epistomal setae; np: nasal plate; apm: apical margin), (D) head, ventral view (prm: prementum; os: occipital suture; atp: anterior tentorial pit; ptp: posterior tentorial pits; gs: gular suture), (E) Mississippi, Washington County (CWOC0045), dorsal view with deciduous processes (dp); (F) Texas, Brooks County (CWOC0002) dorsal, scale bar $1 \mathrm{~mm}$; (G) Texas, Mason Co. (TTU-Z_219308), dorsal view with deciduous processes (dp). 
Rostrum. Only very slightly wider than long (Fig. 4A, E-G), anteriorly slightly widened apicad of antennal insertion; shape in cross-section subrectangular. In dorsal view (Fig. $4 A, E-G$ ), outline of rostrum subquadrate; dorsolateral margins (Fig. 4A, dlm; dorsal margin of scrobe in dorsal view) arcuate, anteriorly and posteriorly diverging, elevated (see Fig. 4C); apical margin deeply emarginate, somewhat sinuate (Fig. 4C, apm). Nasal plate (Fig. 4C, np; see Vaurie 1963, Girón and Howden 2019) moderate in size, with depressed surface, positioned nearly perpendicular to surface of epistoma, with posterior margin bluntly elevated. Epistoma (Fig. 4C, ep) distinctly depressed, with small, oval scales along posterolateral areas; posterior margin of epistoma transversely elevated. Epistomal setae (Fig. 4C, eps) moderately thick, long and dense, becoming longer apically, apicomedially pointing. Dorsal surface of rostrum with one deep, median sulcus (Fig. 4C, ms), anteriorly broadly widened forming large, elongate triangular depression, extending from posterior margin of epistoma to midpoint between eyes, reaching and somewhat fusing with median frontal fovea; with two short dorsolateral sulci (Fig. 4C, ds) positioned along posterior half of rostrum; ventrolateral margins (Fig. 4A, vlm) slightly arcuate, anteriorly diverging. Ventral margin of antennal scrobe, often fully visible in dorsal view of rostrum. Rostrum in lateral view (Fig. 4B) slightly and gradually expanded apically, with dorsal outline nearly straight along basal half; occipital suture (Fig. 4B, D, os) extending from anteroventral margin of eye to ventral mid-length of rostrum to meet anterior tentorial pit (Fig. 4D, atp), continuing anteriorly to near apex of rostrum; margins of mandibular incision with rather long, thick suberect setae (Fig. 4D); gular sutures (Fig. 4D, gs) basally fused together medially, separating at base of rostrum, extending to posterior tentorial pits (Fig. 4D, ptp). Antennal insertion near anterior third of rostrum. Scrobe lateral (Fig. 4B), slightly arcuate, gradually and slightly expanded and shallower towards eye, initiating in apicodorsal region, terminating in basimedial region of rostrum, with dorsal margin welldefined throughout, ending at mid-length of anterior margin of eye; ventral margin of scrobe ending near mid-length of rostrum; scrobe covered with scales along posterodorsal areas (Fig. 4B).

Mouthparts. Mandibles with multiple setae along dorsal, outer and ventral areas surrounding scar (Fig. 4B-D; few scales on outer surface; mandibular scar protruded and sharply raised (Fig. 4C; see also Girón and Franz 2012, character 14); deciduous processes (Fig. 4E, G, dp) large, sickle-shaped, nearly symmetrical, nearly as long as greatest dorsal width of rostrum. Maxillae (Fig. 5A) with cardo (cd) longitudinally twisted; lateral margin of stipes (st) with five or six setae of various lengths along basal half of margin and two along distal region, ventral surface with three thick setae near mesal margin; galeo-lacinial complex (Fig. 5A, ga+la; not considering thick apical setae) extending to apex of maxillary palpomere 2 (Fig. 5A, mpm2), apical region round, mesal margin with basal tuft of very long setae (nearly as long as longest length of cardo) and one lacinial tooth (Fig. 5A, lat), apical region with four to five thick and flattened major galeal teeth (Fig. 5A, gat), accompanied by numerous minor teeth and dense, thick setae of various lengths; palpiger (Fig. 5A, pg) membranous along apical half, basal region more sclerotised, with transverse row of setae along margin of scerotised region of dorsal face; maxillary palps with three palpomeres; maxillary 
palpomere 1 (Fig. 5A, mpm1) nearly 1.5-times longer than 3 (Fig. 5A, mpm3), 2-times longer than 2 (Fig. 5A, mpm2), with oblique apical margin; apicolateral margin of palpomere 1 with one ventral seta; apical margin of palpomere 2 with one dorsal and one ventral seta; palpomere 3 slightly longer than wide, with several longitudinal sulci and a crown of papillae across apical surface. Labium with prementum (Fig. 4D, prm) entirely covering maxillary palps; sub-rectangular, surface reticulate, with apical margin medially slightly angulate; ventral surface basi-medially depressed with long setae along each side; ligula narrow (Fig. 5C, Ig), with one strong long mesal seta at apex; labial palps (Fig. 5B, C) with three palpomeres; labial palpomeres 1 and 3 similar in length (Fig. 5B, C Ipm1, Ipm3, respectively), palpomere 2 (Fig. 5B, C Ipm2) only slightly shorter; apico-ventral margins of palpomeres 1 and 2 each with one seta, palpomere 3 (Fig. 5B, C Ipm3) slightly longer than wide, with basiconical sensillae across apical surface.

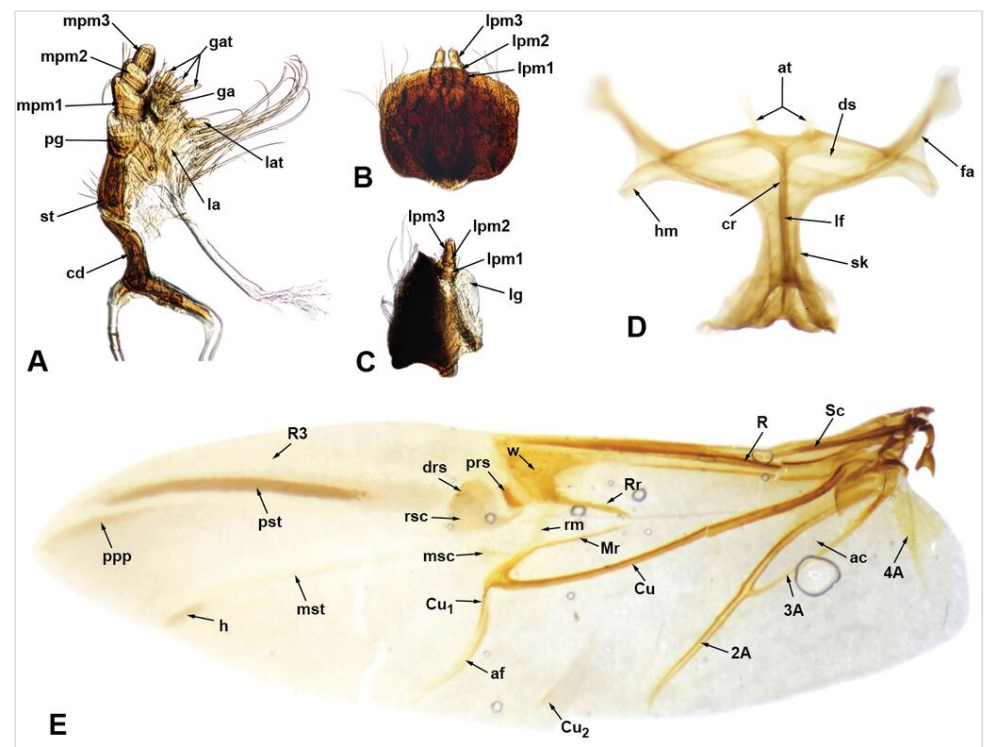

Figure 5. doi

Morphological features of Compsus auricephalus (Louisiana, Acadia Parish): (A-C) mouthparts: (A) right maxilla, ventral view (cd: cardo; st: stipes; pg: palpiger; mpm1: maxillary palpomere 1; mpm2: maxillary palpomere 2; mpm3: maxillary palpomere 3; gat: galeal teeth; ga: galea; lat: lacinial tooth; la: lacinia), (B-C) prementum, (B) dorsal view, (C) lateral view (Ipm1: labial palpomere 1; Ipm2: labial palpomere 2; Ipm3: labial palpomere 3; Ig: ligula), (D) metendosternite, posterior view (sk: stalk; fa: furcal arm; cr: crux; ds: distal sheath; at: anterior tendons; If: longitudinal flange; hm: hemiductus), (E) hindwing (Sc: subcosta; R: radial; Rr: radial recurrent; rm: crossvein between radial system and Mr; Mr: medial recurrent; w: radial window; prs: proximal radial sclerite; rsd: distal radial sclerite; rsc: radial sclerotisation; msc: medial sclerotisation; $\mathrm{Cu}$ : cubital; $\mathrm{Cu} 1$ : branch of $\mathrm{Cu}$; af: apical fold; $\mathrm{Cu} 2$ : branch of $\mathrm{Cu} ; 2 \mathrm{~A}$ : anal vein 2; 3A: anal vein 3; ac: anal cell; 4A: anal vein 4; R3: radial 3; pst: postradial stripe; ppp: posterior part of postradial stripe; $h$ : apical hook of medial stripe; mst: medial stripe). 
Antennae. With 12 antennomeres (Fig. 4A, B). Scape slightly bent along mid section, extending beyond posterior margin of eye, not reaching anterolateral margin of prothorax, passing over ventral fifth of eye (in resting position), densely covered with scales, with sparse, thick, white setae. Funicle with seven antennomeres, 1.3-times longer than scape; funicular antennomeres clavate, progressing from distinctly to slightly elongate; funicular antennomere 2 slightly longer than 1; 1 slightly longer than 3; 4 and 5 similar in shape, shorter than 3; 6, 7 and 8 similar in shape, shorter than 5 . Club with four distinct visible antennomeres (terminal annulation fully differentiated, see Fig. 4B), nearly as long as funicular antennomeres 5-7 combined, nearly 3-times longer than wide, densely covered by translucent setae; club antennomeres gradually decreasing in length towards apex.

Thorax. Pronotum somewhat trapezoid (Fig. 3A), 1.3-times wider at base than apex, 1.2-times wider at base than long; pronotum nearly 0.3 -times length of elytra, with greatest width at base; dorsolateral margins anteriorly converging, posteriorly subparallel; posterior margin only very slightly bisinuate, with lateral areas depressed, and covered by densely arranged plumose setae. Dorsal surface of pronotum medially flattened to depressed, with dorsolateral, longitudinal, broad elevations, irregularly foveate to punctate; lateral surfaces flattened, irregularly foveate to punctate; scales appressed, evenly and densely distributed for the most part; with scattered recurvate setae, anteriorly or medially directed; median area of pronotum often posteriorly depressed. Prothorax in lateral view (Fig. 3B) subquadrate to trapezoid; anterior margin straight and slightly oblique, without postocular lobe, fringed with angulate scales and sometimes with plumose setae projecting anteriorly; postocular vibrissae absent; dorsolateral surface broadly and longitudinally elevated posteriad coxal insertion; posterolateral surface depressed. Metascutum partially covered with plumose scales, especially along posterior margin. Scutellar shield subquadrate to elongate with round corners, covered by scales. Prosternum (Fig. 3C) slightly longer than mesosternite, similar in length to metasternite; prosternum with transverse anterior and posterior sulci; procoxal cavities contiguous, 0.7-times closer to anterior than to posterior margin of prosternum; anterior margin of prosternum fringed by regular setae, plumose setae and scales. Mesosternite (Fig. 3C) with intercoxal process slightly elevated, with recurvate setae; mesocoxal cavities separated by distance nearly 0.3 -times greatest width of each mesocoxal cavity. Metasternite (Fig. 3C) with median sulcus short, shallow, Y-shaped, positioned near posterior margin; metacoxal cavities separated by distance nearly 0.8-times width of each mesocoxal cavity. Metendosternite (Fig. 5D) with stalk (sk) 1.5-times longer than each furcal arm (fa); stalk somewhat triangular (wider at junction with sternum, gradually narrowing towards crux (cr)); distal sheaths (ds) well developed, as wide as widest point of crux; anterior tendons (at) inserted at dorsal margin of distal sheaths, at mesal one-third between longitudinal flange (If) and furcal arms; furcal arms somewhat triangular, diverging, with apex slightly explanate; hemiducti $(\mathrm{hm})$ well developed, ventrolaterally pointing; posterior mesal sheath extending between dorsal margin of distal sheath and mid-length of stalk. 
Legs. (Fig. 3B, C, F) Prothoracic legs slightly longer than mesothoracic legs, slightly shorter than metathoracic legs; with evenly spaced whitish, recurvate setae along femora, denser along tibiae, especially along antero-ventral margin. Profemur nearly 1.5-times longer than prothorax; profemur gradually enlarging to slightly apicad of midlength, with ventral margin basally straight, distally strongly bisinuate; unarmed, but surface ventrally slightly projected at profemoral expansion. Protibia slightly longer than profemur, slender, apically slightly expanded, with ventral margin slightly arcuate, especially along apical fourth; ventral margin without cuticular teeth, with row of translucent to whitish spiniform setae; protibial apex with anterior margin slightly arcuate, densely fringed by fine setae; mucro shorter than to similar in length to tarsal claw, surpassed by tuft of fine, long setae. Protarsomeres 1 and 3 similar in length, protarsomere 2 0.6-times length of protarsomere 1; protarsomere 5 slightly longer than 3. Mesofemur slightly longer than mesotibia, similar to prothoracic legs; mesotarsomere 1 slightly longer than 3, 3 slightly longer than 2, 5 slightly longer than 1 . Metafemur gradually enlarging towards second third. Metatibia straight, expanded at apical region; metatibial apex with anterior margin obliquely truncate, posteriorly ascending by one third of apical width; setal comb with setae similar in length along apex, longer along ascending region; articular surface (Fig. 3D, as; surrounding tarsal condyle) densely and completely covered with scales; both inner flange (Fig. 3D, if) and outer bevel (Fig. 3D, ob) fringed by row of spines ("corbel enclosed"). Tarsal claws paired, separate, simple.

Elytra. (Fig. 3A, B) 1.6-1.8-times longer than greatest width; greatest width near midlength in males, near posterior third in females; anterior margins jointly similar in width to posterior margin of pronotum, recessed along median third (projected along striae 14, corresponding to emargination of posterior margin of pronotum); humeri oblique, slightly produced; joint elytral width at humeri nearly 1.5-times wider than joint anterior elytral margin, slightly narrower than maximum width of elytra; lateral margins straight and slightly diverging along anterior half to two-thirds, then evenly curved and gradually converging along posterior third; posterior margins each rounded, forming right angle (Fig. 3D). Elytra in lateral view (Fig. 3B) with dorsal outline subplane to slightly convex along anterior half; posterior declivity gradual, evenly convex. Elytra with longitudinallyaligned punctures forming nine complete striae and one incomplete stria (striae 9 and 10 (Fig. 3B, s9, s10) fused near metacoxal insertion); striae narrower than interstriae; punctures separated by distance nearly twice longer than width of each puncture; each puncture with one scale or mostly covered by scales; interstriae 3, 5, 7 (Fig. 3D, i3, i5, i7) and 9 (Fig. 3B, i9) convex along most or part of their length; interstriae 1, 2, 4, 6 and 8 flat to only slightly convex, 10 strongly convex at level of metaventrite; setae similar in length to width of interstriae, recurvate, evenly distributed along interstriae, slightly denser along apical areas; scales covering entire integument, oval, overlapping, uniformly coloured.

Hind wings. (Fig. 5D) Fully developed, elongate, nearly 1.4-times longer than elytra, nearly 3.4-times longer than wide; costal margin slightly sinuate along basal half, evenly and broadly curved thereafter; apex narrowly rounded, posterior margin broadly 
rounded along proximal third; anal lobe not demarcated by emargination of posterior margin of wing. Alar venation well developed: subcosta $(\mathrm{Sc})$, radial $(\mathrm{R})$ and radial recurrent $(\mathrm{Rr})$ well defined, proximal radial sclerite (prs) well developed, darkened, distal radial sclerite (drs) reduced; radial window $(w)$ strongly reduced; radial 3 (R3) very weakly defined (as translucent line), almost reaching alar margin; post radial stripe (pst) darkened, well-defined, almost reaching alar margin; posterior part of postradial stripe (ppp) extending along apical region of wing, nearly one third length of pst, almost reaching alar margin; apical hook of medial stripe (h) conspicuous, nearly one third length of ppp, almost reaching alar margin; medial recurrent $(\mathrm{Mr})$, cubital $(\mathrm{Cu})$ and branch 1 of $\mathrm{Cu}$ (Cu1) well defined; crossvein between radial system (rm) very weakly defined; radial sclerotisation ( $\mathrm{rsc}$ ) and medial sclerotisation (msc) weakly defined; medial stripe (mst) weakly defined, slightly shorter than pst; apical fold (af) distinct, nearly reaching alar margin; branch 2 of $\mathrm{Cu}(\mathrm{Cu} 2)$ reduced, smaller than $\mathrm{h}$, not reaching alar margin; anal vein $2(2 \mathrm{~A})$ well defined, vanishing near margin; anal vein 3 $(3 A)$ short, reaching mid-length towards margin of wing, fusing with $2 A$ to form anal cell (ac); anal vein 4 (4A) lightly sclerotised, surounded by lightly sclerotised area around base; anal area with margin broadly rounded; posterior margin of hind wing fringed by short and fine setae separated from each other by distance similar to length of one seta, fringe extending to apical fold .

Abdomen. Abdominal ventrites (Fig. 3C, E) densely covered with scales; ventrite 1 nearly as long as thoracic metaventrite, with posterior margin sinuate; abdominal ventrite 2 0.7-times as long as 1 , nearly as long as 5; surface of ventrites 1 and 2 nearly flat in males (Fig. 3E), convex in females (Fig. 3C); ventrite 5 1.7-times wider than long, with broadly rounded apex in males (Fig. 3E), 1.5-times wider than long, with rounded apex in females (Fig. 3C).

Male terminalia. Tergite 7 1.3-times wider than long, with apical corners broadly rounded and broadly emarginate at apex; posteromedial and marginal regions darkened, with relatively thick setae; basi-medial and basi-lateral areas with fine, appressed spines. Tergite 8 subquadrate, only slightly wider than long, with apical corners broadly rounded and truncate to slightly emarginate at apex, with relatively long and thick setae throughout, except along basal fourth; apical margin in posterior view broadly triangularly emarginate. Sternite 8 (Fig. 6A) composed of two lateral somewhat rhomboid sclerites joined medially by membrane, slightly more sclerotised along distal half, with membranous spiculum relictum (Fig. 6A, spr); surface of each sclerite with somewhat coarse punctation; lacking setae. Sternum 9 (spiculum gastrale; Fig. 6B) including apodeme, 1.5-times longer than median lobe, posteriorly bifurcate, furcal arms (Fig. 6B1) opposed, somewhat lanceolate, margins slightly irregular along distal region, posteriorly diverging; each furcal arm basally broad, oval and coarsely punctate, with somewhat triangular distal region, more strongly sclerotised, roundly angulate at apex. Tegmen (Fig. 6C; gonocoxites I, see Boudinot 2018) with apodeme slightly longer than median lobe; tegminal plate with two elongate projections (Fig. 6C, C1), each nearly 0.1 -times length of apodeme, finely and densely denticulate along distal half. Aedeagus with median lobe (Fig. 6D; penial sclerite, see Boudinot 2018) 4- 
times longer than wide; basi-ventral margin broadly emarginate; lateral margins subparallel, apex broadly oval (Fig. 6F), distance from apical margin of ostium (gonopore) to apex of median lobe slightly longer than greatest width of apical region of median lobe; medial lobe in lateral view (Fig. 6E) broadly curved by about $130^{\circ}$ basad of mid length, approximately 7.7-times longer than greatest width; greatest width uniform along second third, gradually narrowed along distal third. Endophallus (internal sac) with dense, fine, small papillae (Fig. 6D); with elongate, dorsal, mesal endophallite (Fig. 6F, me) at ostium and ventral membranes (Fig. 6F, vm) projecting lateral and distally forming lateral, elongate, oblique and converging sclerotised regions; basal endophallite complex (see Génier 2019) formed by two basilateral (Fig. 6G, ble) and a mesal (Fig. 6G, bme) rod-like endophallites, followed distally by irregular, transversally folded lamina (Fig. 6G, la) and two lateral bars (Fig. 6G, lb), apically expanding, converging and gradually weakly sclerotised. Aedeagal apodemes (gonocoxites II, see Boudinot 2018) nearly as long as median lobe, slender, slightly broader along anterior half, either fused or very closely articulated to median lobe.

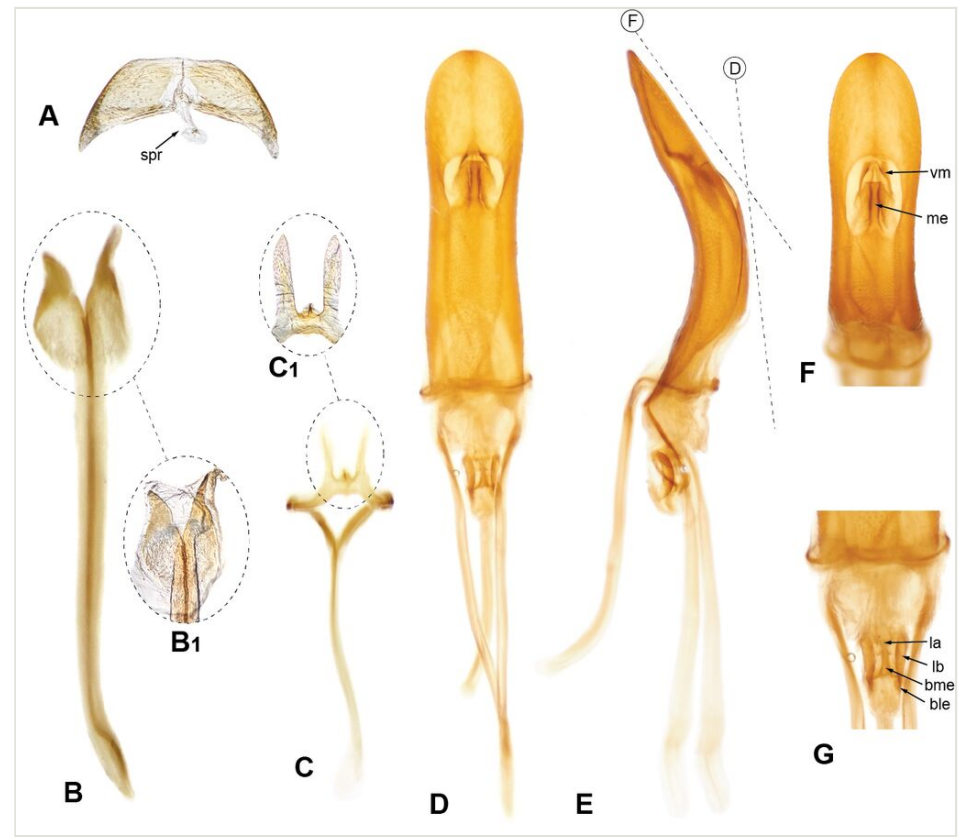

Figure 6. doi

Male genitalia of C. auricephalus: (A) abdominal sternite 8 (spr: spiculum relictum), (B) spiculum gastrale, (B1) detail of furcal arms, (C) tegmen, (C1) detail of projections of tegminal plate, (D) aedeagus, dorsal view (see dashed line $D$ in figure $5 E$ ), (E) aedeagus, lateral view with dashed lines $D$ and $F$ indicating plane for figures $D$ and $F$, respectively, $(F)$ apical region of aedeagus, dorsal view (see dashed line $\mathrm{F}$ in figure $5 \mathrm{E}$; me: mesal endophallite, vm: ventral membranes), $(G)$ detail of basal endophallite complex, dorsal view (la: distal irregular lamina, lb: distal lateral bars), bme: basimesal endophallite, ble: basilateral endophallites). 
Female terminalia. Tergite 7 1.5-times longer than wide, roughly obovate, with anterior and posterior margins broadly and uniformly round, posterior margin more narrowly so; surface sparsely covered by setae, relatively thicker along apical margin; basi-lateral areas with fine, appressed spines. Tergite 8 greatest length 1.2-times longer than greatest width, with anterior margin mesally deeply emarginate; surface gradually more coarsely punctate towards apex, each puncture bearing one seta, setae shorter along apical margin. Sternite 8 (Fig. 7A, B) with apodeme nearly 2.7-times longer than lamina; lamina sagittate, medially divided at apex, 1.7-times longer than wide, apical third covered by setae, gradually more dense towards apex; median region of lamina with mesal paired slightly more sclerotised longitudinal regions; lateral areas of lamina bent upwards (Fig. 7B). Coxites cylindrical (not laterally compressed), nearly 1.6-times longer than lamina of sternum 8; dorsal surface of coxites transversally indented at apical third (Fig. 7; indentation (ind) delimits distal coxites (dcx) from proximal coxites $(p c x)$ ); ventral surface of coxites medially longitudinally membranous, areas adjacent to medial membrane darkened; apical margin of coxites in lateral view sinuate, with dorsal section (Fig. 7C, ds) slightly larger and more round in outline than ventral section (Fig. 7C, vs); ventral section of distal coxites with scarce and thick setae; styli (Fig. 7C, sty; 6F) short, tubular, 3-times longer than wide, apically with eight stout setae of two sizes, longer setae pre-apically situated, shorter setae at apex; styli inserted on dorsal lobe of coxites. Genital chamber slightly longer than sternum 8 including apodeme, with a pair of oval, laminar and concave sclerotisations (Fig. 7C, scl) basad of coxites. Membranes of bursa copulatrix (Fig. 7D, bc) papillate. Spermatheca (Fig. 7D, spe; 6E) hookshaped; ramus (Fig. 7E, ra) longer than collum (Fig. 7E, cl), both apically truncate; corpus (Fig. 7E, crp) shorter and nearly 1.4-times wider than cornu (Fig. 7E, cn); cornu broadly arcuate, apically roundly acute.

Variation: Beyond variation in colouration (two colour morphs: green with green with pink/coppery head and legs or completely white to pale brown; see Figs 1,3) and size $(8-15 \mathrm{~mm})$, the morphology of $C$. auricephalus is fairly homogeneous across the distributional range of the species in the U.S. The morphology of both the male (Fig. 6) and female (Fig. 7) genitalia is also highly conserved within the species with no noticeable differences between colour morphs, which confirms their conspecificity.

Champion (1911) highlighted that specimens from southern populations (e.g. Southern Mexico (Acapulco), Guatemala, Costa Rica, Nicaragua and Panama) were uniformly white, had a more elongate body, more produced and acuminate elytral apices and strongly costate alternating interstriae. We studied specimens with these features (Fig. 8A-B) from Costa Rica, Guatemala, Honduras, Nicaragua and Panama, including specimens studied by Champion himself; we also dissected three specimens from Panama (Fig. 8C-E). In general, the specimens, considered by Champion as a variety of $C$. auricephalus, are externally similar to the northern white form and have overall similar male genitalia, but differ clearly by the following characters: brighter white colouration (Fig. 8A, B; as opposed to pearly white to pale brown), cylindrical pronotum (Fig. 8A; as opposed to trapezoid in C. auricephalus, see Fig. 3A), conspicuously strongly costate interstriae (in comparison with moderately elevated in 
C. auricephalus), angulate elytral declivity (Fig. 8B; as opposed to broadly rounded in C. auricephalus, see Fig. 3B) and acuminate elytral apices (Fig. 8B; as opposed to rounded in $C$. auricephalus, see Fig. 3B). Furthermore, there are evident differences in the shape of the median lobe: median lobe pre-apically widened (Fig. 8C; as opposed to parallel-sided throughout as in C. auricephalus, Fig. 6D); in lateral view, median lobe weakly and rather evenly curved (Fig. 8D; as opposed to broadly and strongly curved basad of mid length, in $C$. auricephalus, Fig. $6 \mathrm{E}$ ), greatest width uniform along median three-fifths (Fig. 8D; as opposed to greatest width uniform along second third, in $C$. auricephalus, Fig. 6E); in dorsal view, gonopore pear-shaped (Fig. 8C; as opposed to oval, as in C. auricephalus, Fig. 6D); basal endophallite complex with different configuration (compare Fig. 8E to Fig. 6G).

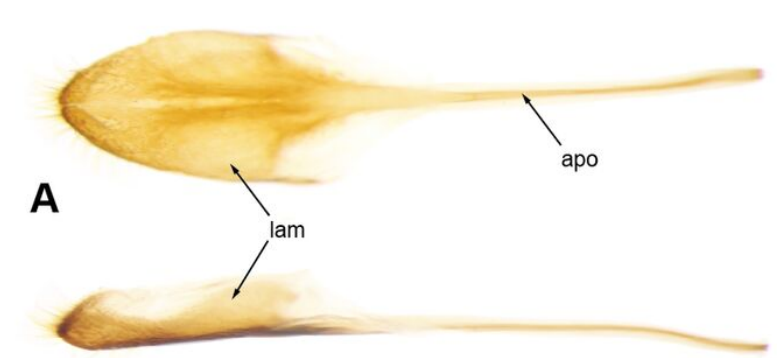

B
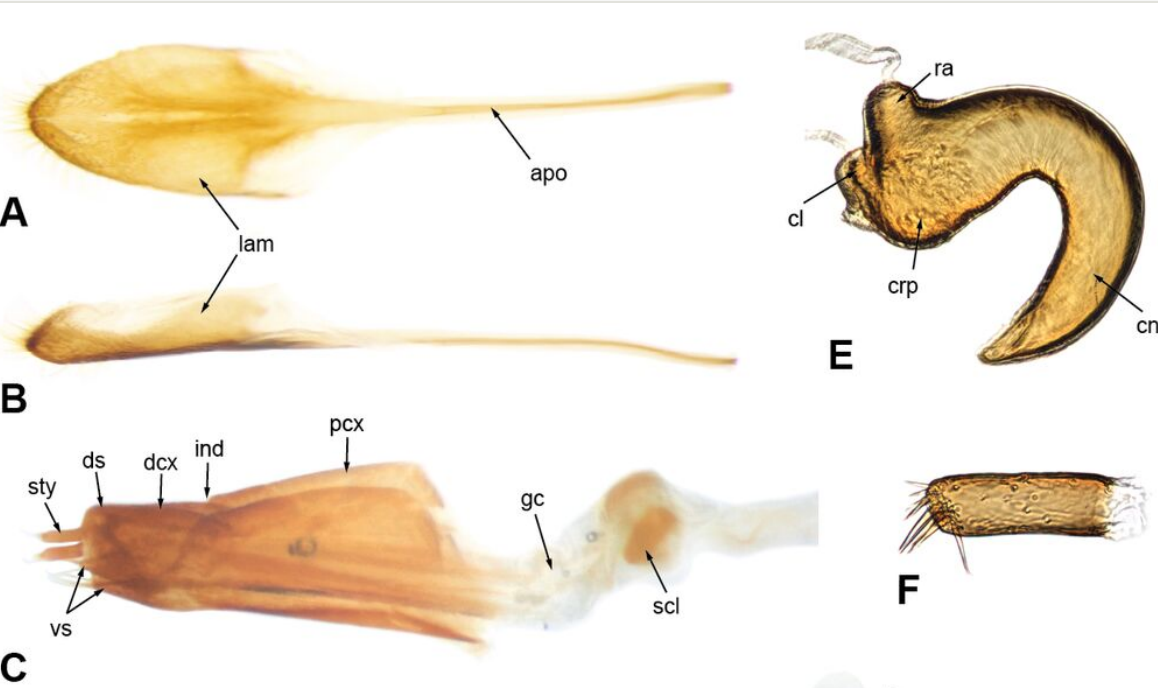

C

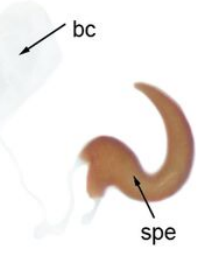

D
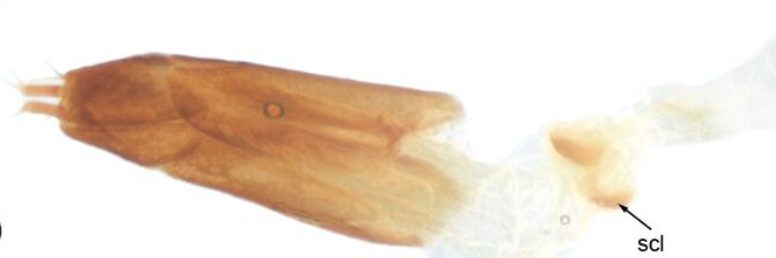

Figure 7. doi

Female genitalia of $C$. auricephalus: (A-B) abdominal sternite 8 (apo: apodeme; lam: lamina), (A) ventral view, (B) lateral view, (C-D) ovipositor, (C) coxites, lateral view (sty: stylus; vs: ventral section of distal coxites; ds: dorsal section of distal coxites; dcx: distal coxites; ind: indentation; pcx: proximal coxites; gc: genital chamber; scl: sclerites) (D) dorsal view (scl: sclerites; bc: bursa copulatrix; spe: spermatheca), (E) spermatheca (ra: ramus; cl: collum; crp: corpus; cn: cornu), (F) detail of stylus. 

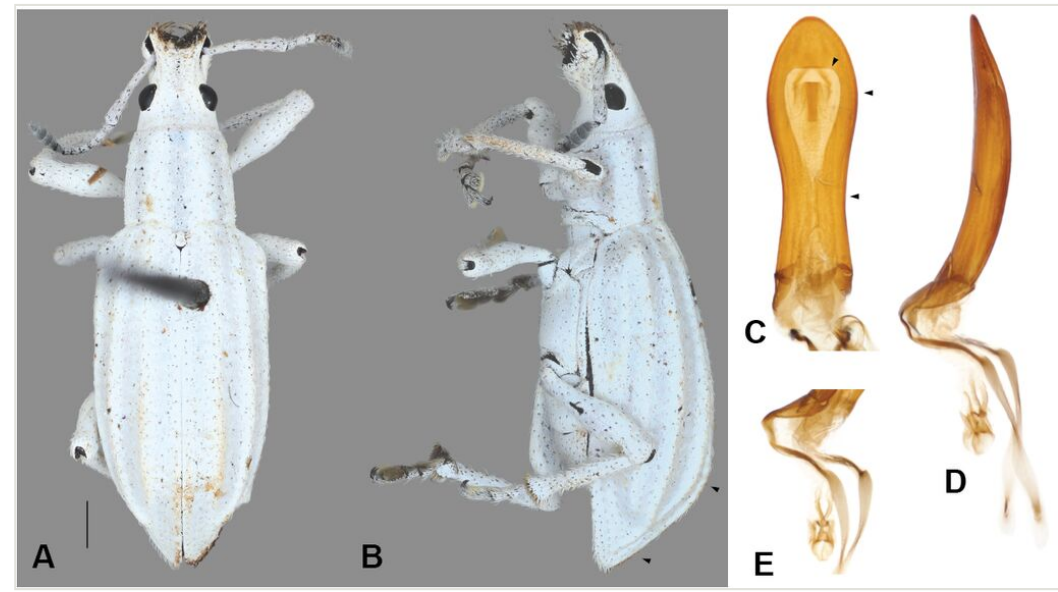

Figure 8. doi

Compsus aff. auricephalus, Panama, Cerro Campana: (A-B) habitus, scale bar $1 \mathrm{~mm}$ : (A) dorsal, (B) lateral, (C) median lobe, dorsal view, (D) aedeagus, lateral view, (E) detail of basal endophallite.

Based on this character combination (external and male genitalia), we conclude that Compsus auricephalus proper extends as far south as Zacapa, Guatemala (CMNEN00019741) and specimens of the Champion (1911) series housed at the BMNH from Costa Rica (Boca del Limón - BMNH(E)1722205; Cangrejal del Aserri $\mathrm{BMNH}(\mathrm{E})$ 1722203), Guatemala (Vera Paz - BMNH(E)1722199); Nicaragua (Chontates - BMNH(E)1722202), Panama (Peñas Blancas - BMNH(E)1722207; $\mathrm{BMNH}(\mathrm{E}) 1722197$; Caldera - $\mathrm{BMNH}(\mathrm{E}) 1722204)$ do not correspond to Compsus auricephalus, but to a different species of Compsus that may or may not be described with a different name. The Acapulco, Mexico specimen, identified by Champion as part of this variety of $C$. auricephalus $(B M N H(E) 1722200)$, is here confirmed to belong to $C$. auricephalus proper. Specimen codes and associated data can be found in Suppl. material 1.

Until more studies can be performed on a broader representation, both across the geographic range and number of specimens of Champion's variety, along with a revision of a larger sample of Compsus species, we refer to this variety as Compsus aff. auricephalus as defined by Champion (1911) and refrain from naming it as new, given that, even though we have made comparisons with some similarly-looking species, we have not seen all the white species of Compsus, nor have we been able to dissect the ones we have examined externally.

\section{Distribution}

The distributional information for $C$. auricephalus was summarised by O'Brien and Wibmer (1982). The distributional data gathered here are mapped in Fig. 9. The updated distribution is as follows: 


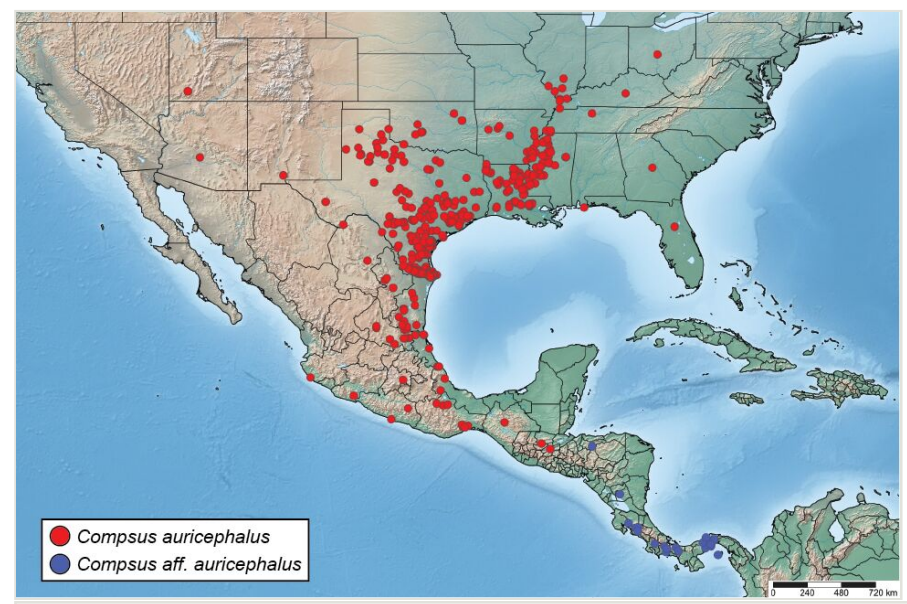

Figure 9. doi

Map of localities recorded for Compsus auricephalus and Compsus aff. auricephalus.

Compsus auricephalus: Guatemala, Mexico, U.S.A.: Alabama (new record), Arizona (new record), Arkansas, Colorado, Florida (new record; one specimen with incomplete data: just "FLA." on label, USNM, needs confirmation), Georgia (one specimen with incomplete data: just "GA" on label, USNM, needs confirmation), Illinois (Blatchley 1925 [overlooked by O'Brien and Wibmer 1982]), Kentucky (new record), Louisiana, Mississippi, New Mexico (new record), Ohio (new record; incomplete data: just "Ohio" on label, USNM, needs confirmation), Oklahoma (Fenton 1944 [overlooked by O'Brien and Wibmer 1982]), Tennessee (new record), Texas, Utah (new record). Canada: Ontario (intercepted at port of entry; McNamara 1991).

Compsus aff. auricephalus: Costa Rica, Honduras (new record), Nicaragua, Panama.

Comments on current distribution of Compsus auricephalus. According to our specimen examination, C. auricephalus occurs north of Zacapa, Guatemala, as far west in the U.S. as Cedar City, Utah and Phoenix, Arizona, as far north as Ohio and as far east to Georgia and Florida. Most of the records are concentrated around Brownsville, Texas and along the Mississippi River. Across the U.S.A., the distribution of $C$. auricephalus seems to be associated with commodities such as cotton plantations (see https://en.wikipedia.org/wiki/Cotton_production_in_the_United_States). There seems to be no geographic pattern of green/white colour morphs.

Records for Florida, Georgia and Ohio (eastern outliers) are represented by single specimens with minimal information: "FLA.", "Ga." and "Ohio", respectively. We report these records here, but highlight that they need confirmation, as these might be cases of interceptions at ports of entry (e.g. Florida), incomplete information (e.g. there is an "Ohio" locality in Hamilton Co., Texas, which is in the area where the species has been recorded) or just mislabelling. 
Western outliers (Arizona, Colorado, New Mexico and Utah) are represented by either records from literature (see O'Brien and Wibmer 1982) or single specimens in collections. Given that there are unique records for each of these occurrences and, even though they have more detailed collecting information (see GBIF dataset at https://doi.org/10.15468/dl.rat633 and Suppl. material 1), they may be adventitious (e.g. transported with plants from elsewhere) and not part of actual established populations of the species in those regions. Data from local collections, as well as more sampling from those areas, would be required to confirm the presence of $C$. auricephalus in those States.

As for Compsus aff. auricephalus, it is known to occur from Yoro, Honduras, south to the Canal Zone in Panama (Fig. 9).

\section{Ecology}

Compsus auricephalus has been collected in palmetto thickets and woods (Blatchley and Leng 1916) and, according to label data, on vegetation along roads, on prairies and using a variety of methods (beating, blacklights, flight intercept traps, malaise traps, pitfall traps, sweeping, manual capture). The species has been found at elevations from sea level up to $1200 \mathrm{~m}$, with most specimens collected below $100 \mathrm{~m}$.

Associated plants: Host plant records were recovered from literature, as well as from label data from specimens in collections. Host specificity in broad-nosed weevils is difficult to assess, given that the presence of adults on a particular plant does not necessarily mean that feeding occurred (Kissinger 1964). A summary including 46 plant species in 23 families is presented in Table 1.

\section{Table 1.}

Host plants associated with Compsus auricephalus. Acronyms in the References column correspond to collections presented in Materials and Methods. Localities with an asterisk $\left({ }^{*}\right)$ correspond to Compsus aff. auricephalus, as defined by Champion (1911).

\begin{tabular}{|l|l|l|l|}
\hline Plant family & Plant species & Locality & References \\
\hline Amaranthaceae & Beta vulgaris[Beet] & Texas & USNM \\
\hline Asteraceae & Ambrosia & Texas & $\begin{array}{l}\text { Mitchell and Pierce 1911 } \\
\text { Champion 1911, USNM }\end{array}$ \\
\hline & $\begin{array}{l}\text { Ambrosia trifida } \\
\text { [Giant ragweed] }\end{array}$ & Texas & USNM \\
\hline & $\begin{array}{l}\text { Baccharis neglecta[False } \\
\text { willow] }\end{array}$ & Texas & USNM \\
\hline & Helianthus[Sunflower] & Mexico (Nuevo & USNM \\
\hline & León) & \\
\hline
\end{tabular}




\begin{tabular}{|c|c|c|c|}
\hline Plant family & Plant species & Locality & References \\
\hline & $\begin{array}{l}\text { Parthenium } \\
\text { hysterophorus } \\
\text { [Santa Maria feverfew] }\end{array}$ & $\begin{array}{l}\text { Mexico } \\
\text { (Coahuila, } \\
\text { Nuevo León) }\end{array}$ & $\begin{array}{l}\text { Soto-Hernández and Barros-Barrios } 2018 \\
\text { USNM }\end{array}$ \\
\hline & $\begin{array}{l}\text { Xanthium } \\
\text { [Cocklebur] }\end{array}$ & Mississippi & MEM \\
\hline Boraginaceae & $\begin{array}{l}\text { Ehretia anacua } \\
\text { (formerly Ehretia elliptica) } \\
\text { [Knockaway] }\end{array}$ & Texas & Blatchley and Leng 1916 \\
\hline \multirow[t]{2}{*}{ Cactaceae } & Opuntia leptocaulis & Texas & USNM \\
\hline & Opuntia lindheimeri & Texas & USNM \\
\hline Cannabaceae & $\begin{array}{l}\text { Celtis laevigata } \\
\text { [Sugarberry] }\end{array}$ & Texas & USNM \\
\hline Cornaceae & $\begin{array}{l}\text { Cornus sericea } \\
\text { [Red-osier dogwood] }\end{array}$ & Texas & USNM \\
\hline Euphorbiaceae & $\begin{array}{l}\text { Ricinus communis } \\
\text { [Castor bean] }\end{array}$ & Texas & USNM \\
\hline \multirow[t]{12}{*}{ Fabaceae } & Acacia & Texas & $\begin{array}{l}\text { Mitchell and Pierce } 1911 \\
\text { Champion 1911, USNM }\end{array}$ \\
\hline & $\begin{array}{l}\text { Acacia farnesiana } \\
\text { [Sweet acacia] }\end{array}$ & Texas & USNM \\
\hline & \multirow{2}{*}{$\begin{array}{l}\text { Baptisia nuttalliana } \\
\text { [Nuttall's wild indigo] }\end{array}$} & Louisiana & MEM \\
\hline & & Texas & VanDyk et al. 2020 \\
\hline & $\begin{array}{l}\text { Lespedeza } \\
\text { [Bush clovers] }\end{array}$ & Tennessee & USNM \\
\hline & $\begin{array}{l}\text { Leucaena pulverulenta } \\
\text { [Mexican Leadtree] }\end{array}$ & Texas & USNM \\
\hline & Medicago sativa[Alfalfa] & Oklahoma & USNM \\
\hline & $\begin{array}{l}\text { Phaseolus vulgaris } \\
\text { [Bean foliage] }\end{array}$ & Texas & USNM \\
\hline & Prosopis sp. & Texas & USNM \\
\hline & Prosopis juliflora & $\begin{array}{l}\text { Mexico (San } \\
\text { Luis Potosí) }\end{array}$ & USNM \\
\hline & $\begin{array}{l}\text { Prosopis glandulosa } \\
\text { [Honey Mesquite] }\end{array}$ & Texas & $\begin{array}{l}\text { Headrick } 2014 \\
\text { Museum of Texas Tech University (TTU) } 2020\end{array}$ \\
\hline & $\begin{array}{l}\text { Vigna unguiculata } \\
\text { [Black-eyed pea] }\end{array}$ & Texas & USNM \\
\hline
\end{tabular}




\begin{tabular}{|c|c|c|c|}
\hline Plant family & Plant species & Locality & References \\
\hline Fagaceae & $\begin{array}{l}\text { Quercus } \\
\text { [Oak] }\end{array}$ & Oklahoma & USNM \\
\hline Gelsemiaceae & $\begin{array}{l}\text { Gelsemium sempervirens } \\
\text { [Carolina Jessamine] }\end{array}$ & Mississippi & MEM \\
\hline \multirow[t]{4}{*}{ Juglandaceae } & \multirow{4}{*}{$\begin{array}{l}\text { Carya illinoinensis } \\
\text { [Pecan] }\end{array}$} & Louisiana & USNM \\
\hline & & Mississippi & MEM \\
\hline & & Texas & USNM \\
\hline & & $\begin{array}{l}\text { Mexico } \\
\text { (Coahuila) }\end{array}$ & Soto-Hernández and Barros-Barrios 2018 \\
\hline Lamiaceae & $\begin{array}{l}\text { Monarda citriodora } \\
\text { [Lemon beebalm] }\end{array}$ & Texas & USNM \\
\hline Lauraceae & $\begin{array}{l}\text { Persea americana } \\
\text { [Avocado foliage] }\end{array}$ & Panama* & USNM \\
\hline \multirow[t]{7}{*}{ Malvaceae } & \multirow[t]{6}{*}{$\begin{array}{l}\text { Gossypium } \\
\text { [Cotton] }\end{array}$} & Arkansas & $\begin{array}{l}\text { Agricultural Research Service, United States } \\
\text { Department of Agriculture } 1978\end{array}$ \\
\hline & & Louisiana & USNM \\
\hline & & Mississippi & Johnson and Cora 2018, MEM \\
\hline & & Oklahoma & $\begin{array}{l}\text { Fenton 1944, Agricultural Research Service, } \\
\text { United States Department of Agriculture 1978, } \\
\text { USNM }\end{array}$ \\
\hline & & Tennessee & $\begin{array}{l}\text { Agricultural Research Service, United States } \\
\text { Department of Agriculture } 1978\end{array}$ \\
\hline & & Texas & $\begin{array}{l}\text { Mitchell and Pierce 1911, Champion 1911, } \\
\text { Pierce 1916, Blatchley and Leng 1916, } \\
\text { Agricultural Research Service, United States } \\
\text { Department of Agriculture 1978, USNM }\end{array}$ \\
\hline & Hibiscus & Mississippi & MEM \\
\hline Piperaceae & Piper sp. & Texas & USNM \\
\hline \multirow[t]{4}{*}{ Poaceae } & $\begin{array}{l}\text { Sorghum bicolor } \\
\text { [Sorghum] }\end{array}$ & $\begin{array}{l}\text { Mexico (Nuevo } \\
\text { León) }\end{array}$ & USNM \\
\hline & $\begin{array}{l}\text { Triticum } \\
\text { [Wheat] }\end{array}$ & Oklahoma & USNM \\
\hline & \multirow{2}{*}{$\begin{array}{l}\text { Zea } \\
\text { [Corn] }\end{array}$} & Mississippi & USNM \\
\hline & & Nicaragua & Maes and O'Brien 1990 \\
\hline Polygonaceae & $\begin{array}{l}\text { Rumex crispus } \\
\text { [Curly dock] }\end{array}$ & Texas & USNM \\
\hline
\end{tabular}




\begin{tabular}{|c|c|c|c|}
\hline Plant family & Plant species & Locality & References \\
\hline \multirow[t]{8}{*}{ Rosaceae } & Crataegus[Hawthorn] & Louisiana & USNM \\
\hline & $\begin{array}{l}\text { Fragaria } \times \text { ananassa } \\
{[\text { Strawberry] }}\end{array}$ & Tennessee & USNM \\
\hline & \multirow{2}{*}{$\begin{array}{l}\text { Malus domestica } \\
\text { [Apple] }\end{array}$} & Illinois & Dmitriev 2015 \\
\hline & & Mississippi & MEM \\
\hline & \multirow{3}{*}{$\begin{array}{l}\text { Prunus persica } \\
\text { [Peach] }\end{array}$} & Louisiana & USNM \\
\hline & & Illinois & Dmitriev 2015 \\
\hline & & Mississippi & MEM \\
\hline & $\begin{array}{l}\text { Pyrus } \\
\text { [Pear] }\end{array}$ & Mississippi & MEM \\
\hline \multirow[t]{2}{*}{ Rutaceae } & \multirow[t]{2}{*}{ Citrus } & Texas & Woodruff 1985, Headrick 2014, USNM \\
\hline & & $\begin{array}{l}\text { Mexico (Nuevo } \\
\text { León, } \\
\text { Tamaulipas) }\end{array}$ & USNM \\
\hline \multirow[t]{2}{*}{ Salicaceae } & $\begin{array}{l}\text { Populus } \\
\text { [Cottonwood] }\end{array}$ & Tennessee & USNM \\
\hline & $\begin{array}{l}\text { Populus deltoides } \\
\text { [Eastern cottonwood] }\end{array}$ & Texas & USNM \\
\hline \multirow[t]{2}{*}{ Santalaceae } & $\begin{array}{l}\text { Phoradendron } \\
\text { [Mistletoe] }\end{array}$ & & Champion 1911 \\
\hline & $\begin{array}{l}\text { Phoradendron flavescens } \\
\text { [Mistletoe] }\end{array}$ & Texas & $\begin{array}{l}\text { Mitchell and Pierce 1911, Tucker } 1919 \\
\text { (adventitious), Anderson } 1994 \text { (incidental), } \\
\text { USNM }\end{array}$ \\
\hline \multirow[t]{3}{*}{ Solanaceae } & $\begin{array}{l}\text { Nicotiana repanda } \\
\text { [Fiddleleaf tobacco] }\end{array}$ & Texas & USNM \\
\hline & \multirow{2}{*}{$\begin{array}{l}\text { Solanum } \\
\text { [Potatoes] }\end{array}$} & Mississippi & MEM \\
\hline & & Texas & USNM (on foliage of Irish potatoes) \\
\hline Verbenaceae & $\begin{array}{l}\text { Lantana camara } \\
\text { [Wild sage] }\end{array}$ & Texas & Blatchley and Leng 1916 \\
\hline
\end{tabular}

\section{Biology}

According to Blatchley and Leng (1916), citing Pierce (1916), Compsus auricephalus "lays its eggs in a mass of gummy substance on leaves and folds a portion on the leaf over them so that they are perfectly concealed. [...] The eggs hatch in seven or eight days and the larvae enter the ground to feed on the roots of plants." This behaviour matches Howden's oviposition category 9: eggs and adhesive placed in fabricated or discovered niches, usually without use of rostrum, which is typical of most "Adelognatha" (Howden 1995). "The larvae are typical legless, C-shaped, white to 
translucent grubs that feed on roots in soil [...] adults are collected in June and July in southern Texas (Headrick 2014).

According to Headrick (2014), damage caused by C. auricephalus on Citrus is significant in Texas; most of the damage is associated with the underground larval stages which open the door for Phytophthora (Oomycota) infections to the trees; the habits of the larvae make control measures difficult and costly. In addition, $C$. auricephalus has been reported to damage young cotton plants in Arkansas, Oklahoma, Tennessee and Texas (Agricultural Research Service, United States Department of Agriculture 1978).

As for natural enemies, Tetrastichus compsivorus Crawford, 1914 (Hymenoptera: Chacidoidea, Crawford 1914) has been recorded from eggs of C. auricephalus in Oklahoma (Crawford 1914), as well as from Texas, Mississipi (Krombein et al. 1979) and Nicaragua (Maes and O'Brien 1990). There are mentions of Gray Grosbeak (Pyrrhuloxia sinuata) and Cardinal (Cardinalis cardinalis) feeding on miscellaneous beetles including C. auricephalus (McAtee 1908).

Barcode data: Molecular data from the mitochondrial gene Cytochrome Oxidase subunit I (COI) was obtained for the specimen identified as USNMENT01070595 (see Suppl. material 1). The sequence of $361 \mathrm{bp}$ is identified with the GenBank number MN344151.1 (https://www.ncbi.nlm.nih.gov/nuccore/1770599749).

\section{Taxon discussion}

Due to its variation in size and colouration (two main colour morphs: predominantly green with pink/coppery head and part of the legs or completely white to pale brown), Compsus auricephalus can be confused with a number of eustyline species from Central and South America, not only with other Compsus species, but with species of other genera in the Compsus genus complex in South America and even species in the Exophthalmus genus complex in Central America. Colouration patterns alone partly overlap with at least a dozen different species; in these instances, particularities of the colouration pattern (e.g. colouration of legs and/or elytra) along with characters of the elytral sculpture allow for differentiation. Here, we illustrate and discuss some characters useful for distinguishing $C$. auricephalus from look-alike species.

Compsus adonis Marshall, 1922 (Marshall 1922) is a brightly-coloured species with the head, anterior margin of the pronotum, apical region of elytra and outer surfaces of the tibia covered with pink scales, with the elytra, remainder of prothorax and elytra and femora mostly green; the apices of the femora and the tarsi are covered by blue scales and the elytra have irregular areas covered by white scales blending in with the general green coverage (Fig. 10B). This very particular colouration pattern is quite different from the colourations exhibited by $C$. auricephalus. In addition, the less regularly distributed elytral punctation, along with the irregularly-elevated surface of the elytra and projected elytral apices in $C$. adonis further allows its recognition. Compsus adonis is endemic to Colombia (Wibmer and O'Brien 1986). 


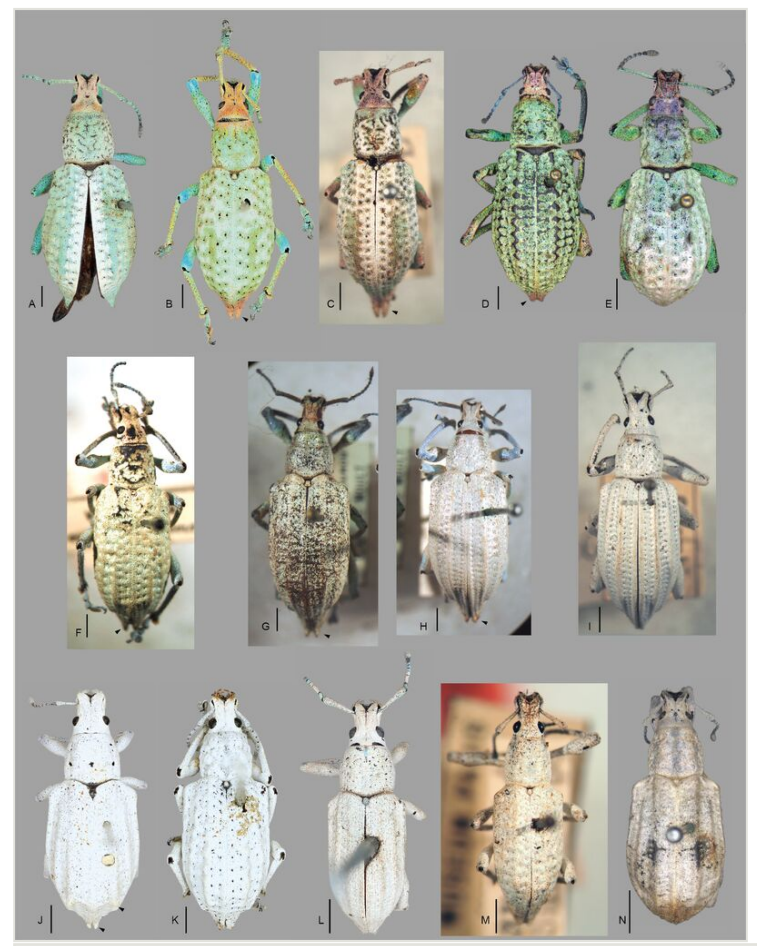

Figure 10. doi

Habitus of species in the Compsus genus complex apparently similar to C. auricephalus: (A) Oxyderces viridipes NHRS-JLKB000022891 (syntype, NHRS), (B) Compsus adonis BMNH(E)1722340 (holotype, NHMUK, (C) Compsus bellus (holotype, MNHN), (D) Oxyderces exaratus (syntype, MNHN), (E) Compsus violaceus (syntype, MNHN), (F) Compsus sulcicollis (holotype, MNHN), (G) Compsus lacteus (MNHN, white form), $(\mathrm{H})$ Compsus lacteus (MNHN, green form), (I) Compsus albus (syntype MNHN), (J) Oxyderces cretaceus ZMUC00037512 (holotype, ZMUK), (K) Oxyderces mansuetus NHRS-JLKB000023017 (paratype, NHRS), (L) Compsus exanguis NHRS-JLKB000022886 (syntype, NHRS), (M) Compsus virginalis (syntype, MNHN) and (N) Oxyderces cinereus (syntype, MNHN). Scale bars: $1 \mathrm{~mm}$.

Compsus albus Hustache, 1938 (Hustache 1938) shares many of the characteristics present in specimens of the white form of $C$. auricephalus, as well as with $C$. aff. auricephalus, as defined by Champion (1911): body covered by uniformly-white scales, costate interstriae 3,5 and 7 , acuminate elytral apices with regularly-aligned elytral punctures. The holotype of this species has a shiny lustre throughout (Fig. 10I). Compsus albus can be differentiated by the lack of a medial sulcus along the rostrum, which is present in C. auricephalus (compare Fig. 10 l vs. Figs 2, 3A, C). Compsus candidus is similar to $C$. albus, both species being endemic to Colombia (Wibmer and O'Brien 1986).

In Compsus bellus Hustache, 1938 (Hustache 1938), the head and distal parts of the legs are covered by coppery/pink scales just as in the green form of $C$. auricephalus. Compsus bellus can be recognised by its combined green and white thorax and elytra, 
where the elytra have a broad white medial longitudinal stripe (Fig. 10C; as opposed to either uniformly-green or uniformly-white thorax and elytra in C. auricephalus, see Figs 1,3 ) and by the strongly-projected elytral apices (Fig. 10C; apex of elytra not projected in C. auricephalus, see Fig. 3A). In addition, there are no conspicuously-elevated interstria along the elytra in C. bellus. Even though $C$. bellus is currently considered a junior synonym of $C$. adonis, by comparing both holotypes, beyond the evident differences in colouration (compare Fig. 108B vs. Fig. 10C), the elytral punctation and the length of the apical elytral projections suggest that these are, in fact, different species, although, until more detailed studies, including dissections of male and female genitalia can be performed, we refrain from making any taxonomic changes at this time. Compsus bellus is endemic to Colombia (Wibmer and O'Brien 1986).

Compsus divisus Hustache, 1938 (Hustache 1938) has the head and entire legs (except the tarsi) covered by pink scales, with bluish-green scales covering thorax and elytra; it also has part of the odd-numbered elytral interstriae elevated. The round eyes in lateral view of the head, along with the presence of an elevated, glabrous and smooth longitudinal ridge along the head and rostrum would allow the recognition of $C$. divisus; this longitudinal ridge suggests that this species may actually be better placed in the genus Exophthalmus (Franz 2012, Zhang and Franz 2015). Compsus divisus is endemic to Colombia (Wibmer and O'Brien 1986).

Compsus exanguis (Boheman, 1833) (Schönherr 1833) is generally similar to the white form of $C$. auricephalus, except for the conspicuous thick setae uniformly distributed all over the surface of the body and the presence of green scales at midpoint of the anterior margin of the pronotum, scattered through the antennae, at the apex of the tibiae and along the tarsi (Fig. 10L). Compsus divisus is endemic to Peru (Wibmer and O'Brien 1986).

Compsus lacteus (Fabricius, 1781) (Fabricius 1781) has variable colour morphs within the same colour range as $C$. auricephalus (i.e. green and white colour forms); however, the head is never pink and the legs and antennae are always distally blue (Fig. 10G, $\mathrm{H})$. Compsus lacteus has strongly-projected eyes, projected elytral apices and conspicuous setae along the surface of the prothorax and elytra, which differentiates it from C. auricephalus. Compsus lacteus is distributed in Brazil, French Guiana, Guadeloupe and Jamaica (O'Brien and Wibmer 1982, Wibmer and O'Brien 1986).

Compsus sulcicollis Hustache, 1938 (Hustache 1938) has the head covered by coppery/pink scales, with the legs covered by light green and blue scales; the thorax and elytra are uniformly covered by light green scales; the surface of elytral interstriae 3 is elevated only along the elytral declivity in $C$. sulcicollis and the apices of the elytra are strongly projected (Fig. 10F); these features allow its differentiation from $C$. auricephalus. Compsus sulcicollis is endemic to Venezuela (Wibmer and O'Brien 1986).

Compsus violaceus Hustache, 1938 (Hustache 1938) follows the general pink head/ green body pattern of the green form of $C$. auricephalus; however, the rostrum of $C$. 
violaceus is covered by pink scales, while the frons and mid-anterior section of the pronotum are covered by purple scales; the remainder of the pronotum and anterior half of the elytra are covered by pale green scales, the posterior half of the elytra are covered mostly by white scales and the legs are covered completely by iridescent green scales (Fig. 10E). In addition, the overall body shape of $C$. violaceus is pyriform (Fig. 10E), as opposed to subrectangular as in C. auricephalus (Fig. 3A). Compsus violaceus is endemic to Colombia (Wibmer and O'Brien 1986).

Compsus virginalis Faust, 1892 (Faust 1892) is generally similar to the white form of $C$. auricephalus and more so to C. aff. auricephalus, as defined by Champion (1911), as it is uniformly covered by white scales, has cylindrical prothorax and acuminate elytral apices. It can be distinguished by its somewhat irregular elytral punctation and irregularly-elevated elytral surface (Fig. 10M; as opposed to having costate oddnumbered elytral interstriae). Compsus virginalis is endemic to Venezuela (Wibmer and O'Brien 1986).

Exophthalmus carneipes Champion, 1911 (Champion 1911), E. cupreipes Champion, 1911 (Champion 1911), E. opulentus Boheman, 1840 (Schönherr 1840) and $E$. vitticollis Champion, 1911 (Champion 1911) are generally similar to the green form of C. auricephalus, all of them being predominantly covered by green scales with the head covered by pink scales. The legs fully covered by pink scales and the presence of an elevated, glabrous and smooth longitudinal ridge along the head and rostrum clearly differentiate C. auricephalus from these Exophthalmus species. In addition, $E$. carneipes, which is distributed in Costa Rica, Honduras and Panama (O'Brien and Wibmer 1982), has transverse bands of pink scales along the elytral shoulders and elytral apices, (see https://www.inaturalist.org/taxa/874484-Exophthalmus-carneipes). On the other hand, E. cupreipes, which is endemic to Mexico (O'Brien and Wibmer 1982), has a more elongated rostrum and its scales are green with golden tones (Bautista-Martínez et al. 2020), very similar to E. opulentus from Guatemala and Mexico (O'Brien and Wibmer 1982); these two species can be differentiated by the fully pink head in E. opulentus (https://www.inaturalist.org/taxa/269236- Exophthalmusopulentus), whereas the head of E. cupreipes has green lateral areas. Exopthalmus vitticollis, from Belize and Guatemala (O'Brien and Wibmer 1982) can be recognised by the presence of a pink median longitudinal stripe along the pronotum (https:// www.inaturalist.org/taxa/1073743-Exophthalmus-vitticollis).

Oxyderces cinereus (Hustache, 1938) (Hustache 1938) is generally similar to the white form of $C$. auricephalus, especially because of its elevated odd-numbered elytral interstriae. It differs by the pyriform shape of the body and the greyish colouration of the scales covering the head and the mid and lateral lines of the pronotum, along with the presence of a pair of relatively-large dark spots about mid-length on the elytra (Fig. $10 \mathrm{~N})$. Oxyderces cinereus is endemic to Ecuador (Wibmer and O'Brien 1986).

Oxyderces cretaceus (Fabricius, 1792) (Fabricius 1792) is similar to the white form of Compsus auricephalus and nearly identical to $C$. aff. auricephalus, as defined by Champion (1911). The sculpture of the pronotum with three basal foveae, the tubercles 
of interstriae 3 at elytral declivity and the apical projections of the elytra clearly distinguish $O$. cretaceus (Fig. 10J). Oxyderces cretaceus has been recorded from Guadeloupe and Martinique (O'Brien and Wibmer 1982). Records of "Compsus auricephalus" in iNaturalist from Trinidad and Tobago (https://www.inaturalist.org/ observations/32103921) may actually correspond to O. cretaceus, but the available specimen photographs do not allow its confirmation.

Oxyderces exaratus (Hustache, 1938) (Hustache 1938) is similar to the green form of Compsus auricephalus, in the presence of pink scales covering the head and green scales covering most of the body. They differ mostly by the presence of blue scales covering the antennae and tarsi and by the rounded projections of the elytral apices which are covered by pink scales (Fig. 10D). In addition, the surface of the elytra is irregularly elevated in $\mathrm{O}$. exaratus, in contrast with the elevated odd-numbered elytral interstriae typical of $C$. auricephalus (see Fig. 3A). Oxyderces exaratus is endemic to Colombia (Wibmer and O'Brien 1986).

Oxyderces mansuetus Hustache, 1938 (Hustache 1938) is similar to the white form of Compsus auricephalus, differing by the deep punctation of both pronotum and elytra, resulting in irregularly-elevated elytral surface, especially along the posterior half of elytra in O. mansuetus (Fig. 10K). In addition, O. mansuetus has strongly projected eyes and acuminate elytral apices (Fig. 10K) and is endemic to Argentina (Wibmer and O'Brien 1986).

Oxyderces viridipes Boheman, 1840 (Schönherr 1840) is a species with a very similar colouration pattern to the green form of Compsus auricephalus, especially because of the pink colouration of the head. The fully green legs and white longitudinal band along the elytral interstriae 1 and 2, along with the sculpture of the elytra lacking elevated interstriae (Fig. 10A), clearly distinguish O. viridipes, which is endemic to Colombia (Wibmer and O'Brien 1986), quite common in Medellín and surrounding areas (see http s://www.inaturalist.org/observations?taxon id=869547). Oxyderces viridipes has been found domestically in Pennsylvania and California (https://www.inaturalist.org/ observations/6246529) and intercepted at south-eastern ports of entry in the U.S.A.

\section{Acknowledgements}

We thank the curators and collection managers who made specimens and data available to us for study: Charles O'Brien, Emmy Engaser, Sangmi Lee, Nico Franz (ASUCOB); Robert Anderson (CMNC); Nicole Gunter (CLEV); Tommy McElrath (INHS); Victoria Bayless, Nathan Lord (LSAM); Terence Schiefer (MEM); Hélène Perrin and Antoine Mantilleri (MNHN); Maxwell Barclay (NHMUK); Johannes Bergsten (NHRS); Luciana Musetti and Norman Johnson (OSUC); Karen Wright (TAMUIC); Floyd Shockley (USNM); Michael Kuhlmann (ZMUK); Alexey Solodovnikov (ZMUC): we are grateful for their patience and flexibility. This project was delayed due to the unprecedented Coronavirus pandemic of 2020. María Guadalupe del Río allowed us to use her photo of the holotype of Platyomus auriceps Schönherr, 1840. We thank Matthew Buffington, Systematic 
Entomology Laboratory, USDA, for taking the necessary images of specimens required to complete this project and Elisabeth Roberts (USDA, SEL) for data entry. Mention of trade names or commercial products in this publication is solely for the purpose of providing specific information and does not imply recommendation or endorsement by the USDA. The USDA is an equal opportunity provider and employer.

\section{References}

- $\quad$ Agricultural Research Service, United States Department of Agriculture (1978) 31st Annual Conference Report on Cotton Insect Research and Control. Dallas, Texas, Jan 9-11. 75 pp. URL: https://books.google.com/books?

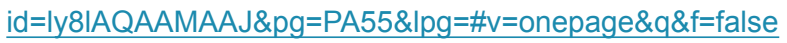

- A.J. Cook Arthropod Research Collection (2020) The Albert J. Cook Arthropod Research Collection, occurrence dataset. https://doi.org/10.15468/mtagq6. Accessed on: 2020-3-25.

- Alonso-Zarazaga M, Lyal CC (1999) A world catalogue of families and genera of Curculionoidea (Insecta: Coleoptera) excluding Scolytidae and Platypodidae . Entomopraxis, Barcelona, Spain.

- $\quad$ Anderson R (1994) A review of New World weevils associated with Viscaceae (mistletoes [in part]) including descriptions of new genera and new species (Coleoptera: Curculionidae). Journal of Natural History 28 (2): 435-492. https://doi.org/ 10.1080/00222939400770201

- $\quad$ Arizona State University Biocollections (2019) Arizona State University Charles W. O'Brien Collection, occurrence dataset. GBIF.org. Release date: 2020-3-25. URL: https://doi.org/10.15468/eeqtx0

- $\quad$ Arizona State University Biocollections (2020) Arizona State University Hasbrouck Insect Collection, occurrence dataset. GBIF.org. Release date: 2020-3-25. URL: https:// doi.org/10.15468/vv6k58

- $\quad$ Bautista-Martínez N, Illescas-Riquelme CP, López-Bautista E, Jones R, López-Buenfil JA (2020) Exophthalmus cupreipes Champion (Coleoptera: Curculionidae) in Citrus Crops in Mexico. Florida Entomologist 102 (4): 708-712. https://doi.org/ 10.1653/024.102.0406

- $\quad$ Blatchley WS, Leng CW (1916) Rhynchophora or weevils of North Eastern America. Nature Publishing Company, 700 pp. https://doi.org/10.5962/bhl.title.122480

- Blatchley WS (1925) Notes on the Rhynchophora of Eastern North America with descriptions of new species, III. Journal of the New York Entomological Society 33 (2): 87-113. URL: https://www.jstor.org/stable/25004076

- Boisduval JBA (1835) Voyage de découvertes de L'Astrolabe exécuté par ordre du Roi, pendant les annies 1826-1827-1828-1829, sous le commandement de M.J. Dumont d'Urville. Faune Entomologique de I'Océan Pacifique, avec illustration des insectes nouveaux recueillis pendant le voyage. Deuxième partie. Coléoptères et autres ordres. J. Tastu, Paris, VII+716 pp. https://doi.org/10.5962/bhl.title.35819

- Boudinot B (2018) A general theory of genital homologies for the Hexapoda (Pancrustacea) derived from skeletomuscular correspondences, with emphasis on the Endopterygota . Arthropod Structure \& Development 47 (6): 563-613. https://doi.org/ 10.1016/j.asd.2018.11.001 
- Callahan M (2019) Auburn University Museum of Natural History Entomology, Auburn University Museum, occurrence dataset. GBIF.org. Release date: 2020-3-25. URL: https://doi.org/10.15468/oo4euw

- $\quad$ Champion GC (1911) Otiorhynchinae Alatae. pp. 178-317. In: Sharp D, Champion GC (Eds) Biologia Centrali-Americana, Volume 4, Part 3. 4(3). Bernard Quaritch, London, 354+15 pt. pp. URL: https://www.biodiversitylibrary.org/page/581653

- $\quad$ Crawford JC (1914) Some new Chalcidoidea . Insecutor inscitiae menstruus 2 (12): 180-182. URL: https://www.biodiversitylibrary.org/page/9141391

- del Río MG, Lanteri A (2003) Revision of the genus Briarius [Fischer de Waldheim] (Coleoptera: Curculionidae). Insect Systematics \& Evolution 34 (3): 281-294. https:// doi.org/10.1163/187631203788964755

- Díaz Batres ME, Comisión nacional para el conocimiento y uso de la biodiversidad (2018) Computarización de la Colección Nacional de insectos Dr. Alfredo Barrera Marín del Museo de Historia Natural de la Ciudad de México, Comisión nacional para el conocimiento y uso de la biodiversidad, occurrence dataset. GBIF.org. Release date: 2020-3-25. URL: https://doi.org/10.15468/ud89ij

- Dmitriev D (2015) Illinois Natural History Survey Insect Collection, Illinois Natural History Survey, occurrence dataset. GBIF.org. Release date: 2020-3-25. URL: https:// doi.org/10.15468/eol0pe

- Evenhuis NL (2020) The insect and spider collections of the world website. http:// hbs.bishopmuseum.org/codens/. Accessed on: 2020-3-25.

- Fabricius JC (1781) Species insectorum, exhibentes eorum differentias specificas, synonyma auctorum, loca natalia, metamorphosin, adjectis observationibus, descriptionibus. 1. Hamburgi \& Kilonii, Bohn, VIII+552 pp. https://doi.org/10.5962/ bhl.title.36509

- Fabricius JC (1792) Entomologia systematica emendata et aucta. Secundum classes, ordines, genera, species adjectis synonimis, locis, observationibus descriptionibus, tom. 1, pars 2. Impensis Christ. Gottl. Proft., Hafniae, 538 pp. URL: https:// www.biodiversitylibrary.org/page/52755487

- Faust J (1892) Reise von E. Simon in Venezuela. Curculionidae. Pars prima. Stettiner Entomologische Zeitung 53 (1-3): 1-44. URL: https://biodiversitylibrary.org/page/ 9266298

- Fenton FA (1944) The insect record for Oklahoma, 1942 and 1943. Proceedings of the Oklahoma Academy of Science 24: 20-25. URL: https://ojs.library.okstate.edu/osu/ index.php/OAS/article/view/3292/2967

- $\quad$ Fischer de Waldheim G (1829) Museum Historiae Naturalis Universitatis Caesareae Mosquensis. Pars II. Insecta. Typis Universitatis Caesareae, Mosquae.

- Franz N (2010) Redescriptions of critical type species in the Eustylini Lacordaire (Coleoptera: Curculionidae: Entiminae). Journal of Natural History 44 (1-2): 41-80. https://doi.org/10.1080/00222930903383495

- $\quad$ Franz N (2012) Phylogenetic reassessment of the Exophthalmus genus complex (Curculionidae: Entiminae: Eustylini, Geonemini). Zoological Journal of the Linnean Society 164 (3): 510-557. https://doi.org/10.1111/j.1096-3642.2011.00774.x

- Gaiger F, Vanin SA (2008) On the utility of female genitalia in the systematics of Curculionidae: examples from Entimini and Hylobiina (Coleoptera: Curculionidae). Annales de la Société entomologique de France (N.S.) 44 (1): 47-57. https://doi.org/ $\underline{10.1080 / 00379271.2008 .10697543}$ 
- GBIF.org (2020) GBIF Occurrence Download. GBIF.org. Release date: 2020-3-25. URL: https://doi.org/10.15468/dl.gl4vo4

- Génier F (2019) Endophallites: a proposed neologism for naming the sclerotized elements of the insect endophallus (Arthropoda: Insecta). Annales de la Société entomologique de France (N.S.) 55 (6): 482-484. https://doi.org/ 10.1080/00379271.2019.1685907

- Germar EF (1824) Insectorum species novae aut minus cognitae, descriptionibus illustratae. Vol. 1. Coleoptera . 1. J. C. Hendelii et filii, Halae. https://doi.org/10.5962/ bhl.title.130964

- Girón J, Franz N (2012) Phylogenetic assessment of the Caribbean weevil genus Lachnopus Schoenherr (Coleoptera: Curculionidae: Entiminae). Invertebrate Systematics 26 (1): 67-82. https://doi.org/10.1071/IS11033

- Girón J, Cardona-Duque J (2018) Estado del conocimiento de los Curculionidae en Colombia. In: Deloya C, Gasca Álvarez HJ (Eds) Escarabajos del Neotrópico (Insecta: Coleoptera). S y G Editores, Mexico, 230+32 pt. pp. [ISBN 978-607-7552-26-0].

- Girón J, Howden AT (2019) Five new species of Pandeleteius Schönherr, 1834 (Coleoptera: Curculionidae: Entiminae: Tanymecini) from South America. The Coleopterists Bulletin 73 (4): 831-845. https://doi.org/10.1649/0010-065X-73.4.831

- Gistel J (1848) Naturgeschichte des Thierreichs für höhere Schulen. Hoffmann, Stuttgart, xvi +216+[4unn.] pp. +32 pl. pp. https://doi.org/10.5962/bhl.title.97235

- Hall D (1995) A revision to the bibliography of the sugarcane rootstalk borer weevil, Diaprepes abbreviatus (Coleoptera: Curculionidae). The Florida Entomologist 78 (2): 364-377. https://doi.org/10.2307/3495911

- Háva J, Rukmane A (2018) Short contribution to the genus Pachyrhynchus from the Philippines (Coleoptera: Curculionidae: Pachyrhynchini). Acta Biologica Universitatis Daugavpiliensis 18 (2): 203-205. URL: http://sciences.Iv/wp-content/uploads/2019/01/ H\%C3\%A1va 218 2.pdf

- Headrick D (2014) 19 Invasive pests: Insects. In: Ferguson L, Grafton-Cardwell EE (Eds) Citrus production manual. Publication 3539. University of California, Agriculture and Natural Resources, Richmond, CA, 433 pp. URL: https://books.google.com/books? $\mathrm{id}=\mathrm{gcH}$ AwAAQBAJ\&pg=PA299\&lpg=PA299\& [ISBN 978-1-60107-840-7].

- Helicon Soft Ltd (2013) Helicon Focus. 5.3.14. URL: https://www.heliconsoft.com/

- Howden AT (1995) Structures related to oviposition in Curculionoidea . Memoirs of the Entomological Society of Washington 14: 53-102.

- Hustache A (1938) Compsus sud-américains (Coleoptera Curculionidae). Bulletin de la Société Entomologique de Belgique 78: 67-118.

- $\quad$ iNaturalist.org (2020) iNaturalist research-grade observations, occurrence dataset. GBIF.org. Release date: 2020-3-25. URL: https://doi.org/10.15468/ab3s5x

- Jekel H (1875) Coleoptera Jekeliana adjecta Eleutheratorum Bibliotheca. Enumeration systématique \& synonymique des Coléoptères européens \& exotiques composant la collection de Henri Jekel. Observations critiques. Descriptions d'Espèces nouvelles. Reproduction ettraduction de genres et espèces publiés dans Des Ouvrages rares Français et Étrangers de manière á former insensiblement la Bibliothèque du Coléoptériste. Livraison 2. Autographie Typomorphe de l'Auteur, Paris, 101-196 pp. URL: https://gallica.bnf.fr/ark:/12148/bpt6k884758k/f3.image 
- Johnson N, Cora J (2018) C.A. Triplehorn Insect Collection (OSUC), Ohio State University. Museum of Biological Diversity, The Ohio State University, occurrence dataset. GBIF.org. Release date: 2020-3-25. URL: https://doi.org/10.15468/efb17f

- Jones RW, Comisión nacional para el conocimiento y uso de la biodiversidad (2018) Captura de datos de la Colección de Curculionoidea (Insecta: Coleoptera) de la Universidad Autónoma de Querétaro, Comisión nacional para el conocimiento y uso de la biodiversidad, occurrence dataset. 1.5. GBIF.org. Release date: 2020-3-25. URL: https://doi.org/10.15468/p4bw2s

- Kissinger DG (1964) Curculionidae of America North of Mexico. A Key to the Genera. Taxonomic Publications, South Lancaster, Massachusetts, vi +143 pp.

- Krombein KV, Hurd PD, Smith DR, Burks BD (1979) Catalog of hymenoptera in America north of Mexico. 1. Smithsonian Institution Press, Washington, 1198 pp. https://doi.org/ 10.5962/bhl.title.5074

- Lacordaire JT (1863) Histoire naturelle des insectes: Genera des Coléoptères ou exposé méthodique et critique de tous les genres proposés jusqu'ici dans cet ordre d'insectes. Tome Sexième. Contenant la famille des Curculionides. Librairie encyclopédique de Roret, Paris, 637 pp. URL: https://biodiversitylibrary.org/page/ 9375856

- Latreille PA (1802) Histoire naturelle, générale et particulière des crustacés et des insectes. Familles naturelles des genres. Tome troisième. L'Imprimerie de F. Dufart, Paris, 467 pp. URL: https://biodiversitylibrary.org/page/24884921

- Lawrence J, Ślipiński A (2013) Adult morphology. In: Lawrence J, Ślipiński A (Eds) Australian beetles volume 1: morphology, classification and keys. 1. CSIRO Publishing, Collingwood, VIC, 520 pp. [ISBN 9780643097285$]$.

- LeConte J, Horn G (1876) The Rhynchophora of America, North of Mexico. Proceedings of the American Philosophical Society 15 (96): 1-442. URL: https:// www.jstor.org/stable/982412

- $\quad$ Marvaldi A, Lanteri A, del Río MG, Oberprieler R (2014) Entiminae Schoenherr, 1823. In: Leschen RAB, Beutel RG (Eds) Handbook of Zoology. Arthropoda: Insecta: Coleoptera, Volume 3: Morphology and systematics (Phytophaga). 3. De Gruyter, Berlin, Germany, 675 pp. [ISBN 978-3-11-027370-0].

- Linnaeus C (1758) Systema Naturae per Regna Tria Naturae: Secundum Classes, Ordines, Genera, Species, Cum Characteribus, Differentiis, Synonymis, Locis. 1. Impensis Direct. Laurentii Salvii, Holmiae, 824 pp. https://doi.org/10.5962/bhl.title.542

- Maes JM, O'Brien C (1990) Lista anotada de los Curculionoidea (Coleoptera) de Nicaragua. Revista Nicaraguense de Entomologia 12: 1-78. URL: http://www.bionica.info/RevNicaEntomo/12-Curculionoidea.pdf

- Marshall GK (1922) On new genera and species of Neotropical Curculionidae. Transactions of the Royal Entomological Society of London 70 (1-2): 181-224. https:// doi.org/10.1111/j.1365-2311.1922.tb02830.x

- Martínez de la Vega G, Comisión nacional para el conocimiento y uso de la biodiversidad (2018) Base de datos de la Colección zoológica del Instituto de Investigación de zonas desérticas de la Universidad Autónoma de San Luis Potosí (BDCZIID-UASLP). Version 1.5. Comisión nacional para el conocimiento y uso de la biodiversidad, occurrence dataset. GBIF.org. Release date: 2020-3-25. URL: https:// doi.org/10.15468/jybhkl 
- Martorell LF (1945) A survey of the forest insects of Puerto Rico, Part II. Journal of Agriculture of Puerto Rico 29 (4): 355-608. https://doi.org/10.46429/jaupr.v29i4.12869

- Marvaldi A, del Río MG, Pereyra V, Rocamundi N, Lanteri A (2018) A combined molecular and morphological approach to explore the higher phylogeny of entimine weevils (Coleoptera: Curculionidae), with special reference to South American taxa. Diversity 10 (3): 1-30. https://doi.org/10.3390/d10030095

- McAtee WL (1908) Food babits of the Grosbeaks. U. S. Department of Agriculture, Bureau of Biological Survey 32: 1-92. https://doi.org/10.5962/bhl.title.54003

- McNamara J (1991) Superfamily Curculionoidea . In: Bousquet Y (Ed.) Checklist of Beetles of Canada and Alaska. 1. Agriculture Canada, Ottawa, vi + 430 pp. URL: http:// www.canacoll.org/Coleo/Checklist/PDF\%20files/CURCULIONIDAE.pdf

- Mitchell JD, Pierce WD (1911) The weevils of Victoria county, Texas. Proceedings of the Entomological Society of Washington 13: 45-62. URL: https:// www.biodiversitylibrary.org/page/2629237

- Morrone JJ (1999) The species of Entiminae (Coleoptera: Curculionidae) ranged in America South of the United States. Anales del Instituto de Biología. Serie Zoología 70 (2): 99-168.

- Museum of Texas Tech University (TTU) (2020) Texas Tech University - Invertebrate Zoology, occurrence dataset. GBIF.org. Release date: 2020-3-25. URL: https://doi.org/ 10.15468/ga4bmd

- $\quad$ Natural History Museum of Utah (UMNH) (2020) Entomology Collection at the Natural History Museum of Utah, occurrence dataset. GBIF.org. Release date: 2020-3-25. URL: https://doi.org/10.15468/iyehoe

- $\quad$ Nichols SW (1989) The Torre-Bueno glossary of entomology. New York Entomological Society \& American Museum of Natural History, New York, N.Y., USA, 840 pp. [ISBN 0-913424-13-7]

- O'Brien C, Wibmer GJ (1982) Annotated checklist of the weevils (Curculionidae sensu lato) of North America, Central America, and the West Indies (Coleoptera: Curculionoidea). Memoirs of the American Entomological Institute 34: 1-382.

- Olivier GA (1807) Entomologie, ou Histoire naturelle des insectes: avec leurs caractères génériques et spécifiques, leur description, leur synonymie, et leur figure enluminée. Coléoptères. Tome Cinquieme. 5. De l'Imprimerie de Baudoin, Paris, 612 pp. [In French]. URL: https://www.biodiversitylibrary.org/page/40641693

- $\quad$ Pascoe F (1881) VI—New neotropical Curculionidae-Part IV. Annals and Magazine of Natural History 7 (37): 38-45. https://doi.org/10.1080/00222938109459470

- Pierce WD (1916) Notes on the habits of weevils. Proceedings of the Entomological Society of Washington 18: 6-10. URL: https://www.biodiversitylibrary.org/page/2570838

- Poole RW, Lewis RE, Gentili P (1996) Nomina insecta nearctica: a check list of the insects of North America. 1. Entomological Information Services, 827 pp. URL: http:// www.nearctica.com/nomina/main.htm

- $\quad$ Prena J (2018) An annotated inventory of the weevils (Coleoptera: Curculionoidea) described by Thomas Say. Bulletin of the Museum of Comparative Zoology 161 (9): 323-401. https://doi.org/10.3099/MCZ161-09.1

- Roberts D (2020) CHAS Entomology Collection (Arctos). Version 13.37. Chicago Academy of Sciences, occurrence dataset. GBIF.org. Release date: 2020-3-25. URL: https://doi.org/10.15468/i5oupp 
- Say T (1824) Descriptions of Coleopterous Insects collected in the late Expedition to the Rocky Mountains, performed by order of Mr. Calhoun, Secretary of War, under the command of Major Long. By Thomas Say, Zoologist to the Expedition. Journal of the Academy of Natural Sciences of Philadelphia 3 (1): 139-216. URL: https:// www.biodiversitylibrary.org/page/24668950

- $\quad$ SCAN (2020) The Symbiota Collections of Arthropods Network (SCAN) serves specimen occurrence records and images from North American arthropod collections. https://scan-bugs.org/. Accessed on: 2020-3-25.

- Schönherr CJ (1823) Curculionides [Tabula synoptica familiae Curculionidum]. Isis von Oken 1823 (10): 1132-1146. URL: https://biodiversitylibrary.org/page/13257284

- Schönherr CJ (1833) Genera et species Curculionidum, cum synonymia hujus familiae. Species novae aut hactenus minus cognitae, descriptionibus a Dom. Leonardo Gyllenhal, C. H. Boheman, et entomologis aliis illustratae, tomus primus, pars prima. Roret, Paris, 681 pp. URL: https://www.biodiversitylibrary.org/page/4108479

- Schönherr CJ (1834) Genera et species Curculionidum, cum synonymia hujus familiae. Species novae aut hactenus minus cognitae, descriptionibus a Dom. Leonardo Gyllenhal, C. H. Boheman, et entomologis aliis illustratae, tomus secundus, pars prima. Roret, Paris, 673 pp. URL: https://www.biodiversitylibrary.org/page/4112093

- Schönherr CJ (1840) Genera et species Curculionidum, cum synonymia hujus familiae. Species novae aut hactenus minus cognitae, descriptionibus a Dom. Leonardo Gyllenhal, C. H. Boheman, et entomologis aliis illustratae, tomus sextus, pars prima. Roret, Paris, 474 pp. URL: https://www.biodiversitylibrary.org/page/4114879

- Schönherr CJ (1842) Genera et species Curculionidum, cum synonymia hujus familiae. Species novae aut hactenus minus cognitae, descriptionibus a Dom. Leonardo Gyllenhal, C. H. Boheman, et entomologis aliis illustratae, tomus sextus, pars secunda. Roret, Paris, 495 pp. URL: https://www.biodiversitylibrary.org/page/4115130

- Segura Ponce de León, R, Comisión nacional para el conocimiento y uso de la biodiversidad C (2020) Computarización de la colección científica del proyecto de control biológico de malezas de CSIRO-Australia. Occurrence dataset. 1.1. GBIF.org. Release date: 2020-6-05. URL: https://doi.org/10.15468/ncaf9t

- Shorthouse DP (2010) SimpleMappr, an online tool to produce publication-quality point maps. www.simplemappr.net. Accessed on: 2020-3-25.

- $\quad$ Simpson SE, Nigg HN, Coile NC, Adair RA (1996) Diaprepes abbreviatus (Coleoptera: Curculionidae): host plant associations. Environmental Entomology 25 (2): 333-349. https://doi.org/10.1093/ee/25.2.333

- $\quad$ Song H, Johnson N (2018) Stuart M. Fullerton Collection of Arthropods (UCFC), University of Central Florida. Version 85.36. Museum of Biological Diversity, The Ohio State University, occurrence dataset. GBIF.org. Release date: 2020-3-25. URL: https:// doi.org/10.15468/kyulwg

- Soto-Hernández M, Barros-Barrios MM (2018) Curculiónidos (Coleoptera: Curculionidae) en el Cultivo de Nogal Pecanero en el Estado de Coahuila, México. Entomología Mexicana 5: 580-586. URL: http://www.entomologia.socmexent.org/ revista/2018/SM/SM\%20580-586.pdf

- Texas A\&M University Insect Collection (2020) Texas A\&M University Insect Collection, occurrence dataset. GBIF.org. Release date: 2020-3-25. URL: https://doi.org/10.15468/ caprah 
- $\quad$ Ting P (1936) The mouth parts of the coleopterous group Rhynchophora . Microentomology 1: 93-114.

- $\quad$ Tucker E (1919) Studies of insects associated with the American Mistletoe. Transactions of the Kansas Academy of Science 30: 143-170. https://doi.org/ $10.2307 / 3624056$

- University of Arizona Insect Collection (2020) University of Arizona Insect Collection, occurrence dataset. GBIF.org. Release date: 2020-3-25. URL: https://doi.org/10.15468/ $\underline{\text { hzkbpg }}$

- Van Dam M, Lam A, Sagata K, Gewa B, Laufa R, Balke M, Faircloth B, Riedel A (2017) Ultraconserved elements (UCEs) resolve the phylogeny of Australasian smurf-weevils. PLOS One 12 (11). https://doi.org/10.1371/journal.pone.0188044

- VanDyk J, Sellers E, Bartlett T (2020) BugGuide - Identification, images, \& information for insects, spiders \& their kin for the United States \& Canada. Version 1.5. United States Geological Survey. Occurrence dataset. GBIF.org. Release date: 2020-3-25. URL: https://doi.org/10.15468/sk2lxk

- Vaurie P (1963) A revision of the South American genus Hyphantus (Coleoptera, Curculionidae, Otiorhynchinae). Bulletin of the American Museum of Natural History 125: 239-304.

- Vega Ortiz, H E, Comisión nacional para el conocimiento y uso de la biodiversidad C (2020) Elaboración de la base de datos de los ejemplares de la colección general de insectos adultos de la Dirección General de Sanidad Vegetal. Occurrence dataset. 1.1. GBIF.org. Release date: 2020-6-05. URL: https://doi.org/10.15468/wajm7f

- Velázquez de Castro AJ (1998) Morphology and taxonomy of the genus Sitona Germar, 1817. (I): the metendosternite (Coleoptera: Curculionidae). Proceedings of the XX International Congress of Entomology.

- Wanat M (2007) Alignment and homology of male terminalia in Curculionoidea and other Coleoptera . Invertebrate Systematics 21 (2): 147-171. https://doi.org/10.1071/ $\underline{\text { IS05055 }}$

- Weissling TJ, Peña JE, Giblin-Davis RM, Knapp Jr. JL (1998) Diaprepes root weevil, Diaprepes abbreviatus (Linnaeus) (Insecta: Coleoptera: Curculionidae). http:// entnemdept.ufl.edu/creatures/citrus/diaprepes root weevil.htm. Accessed on: 2020-3-25.

- Wibmer GJ, O'Brien C (1986) Annotated checklist of the weevils (Curculionidae sensu lato) of South America (Coleoptera: Curculionoidea). Memoirs of the American Entomological Institute 39: 1-563.

- Wolcott GN (1922) Vaquitas de importancia económica en Puerto Rico. Report of the Porto Rico Agricultural Experiment Station, Circular 60: 5-20. URL: https:// books.google.com/books?id=3h9OAAAAYAAJ\&lpg=PA64IA109\&ots=b2SU3ZCPDb\&dq=Vaquitas\%20de\%20importancia\%20econ\%C3\% B3mica\%20en\%20Puerto\%20Rico.\&pg=PA64-IA109\#v=onepage\&q\&f=false

- Woodruff RE (1985) Citrus weevils in Florida and the West Indies: preliminary report on systematics, biology, and distribution (Coleoptera: Curculionidae). The Florida Entomologist 68 (3): 370-379. https://doi.org/10.2307/3495121

- Zerene Systems LLC (2019) Zerene Stacker. T2019-10-07-1410. Release date: 2019-9-10. URL: https://zerenesystems.com/cms/stacker/softwaredownloads 
- Zhang G, Franz N (2015) Systematics of Eustylini - Reclassification of the Exophthalmus genus complex (Curculionidae). https://doi.org/10.13140/RG.

\title{
$\underline{2.1 .2677 .1923}$
}

- Zhang G, Basharat U, Matzke N, Franz N (2017) Model selection in statistical historical biogeography of Neotropical insects-The Exophthalmus genus complex (Curculionidae: Entiminae). Molecular Phylogenetics and Evolution 109: 226-239. https://doi.org/10.1016/j.ympev.2016.12.039

- Zherikhin VV, Gratshev VG (1995) A comparative study of the hind wing venation of the superfamily Curculionoidea, with phylogenetic implications. In: Pakaluk J, Ślipiński A (Eds) Biology, phylogeny, and classification of Coleoptera: papers celebrating the 80th birthday of Roy A. Crowson. 2. Muzeum i Instytut Zoologii PAN, Warszawa, Poland, 1092 pp. [ISBN 978-8385192343].

\section{Supplementary material}

\section{Suppl. material 1: Part of material examined from miscellaneous collections doi}

\author{
Authors: Girón \& Chamorro \\ Data type: Occurrences \\ Brief description: This file contains data of specimens from CLEV, CMNC, INHS, LSAM, MEM, \\ NHMUK and USNM, which are currently not available online. It was created using the IPT \\ template available at https://github.com/gbif/ipt/wiki/occurrenceData. Coordinates were \\ approximated via Google Maps based on locality information.
}

Download file (95.22 kb) 